\title{
Efficacy of topical interventions for temporomandibular disorders compared to placebo or control therapy: a systematic review with meta-analysis
}

\author{
Mariam Mena', Lana Dalbah ${ }^{1,2}$, Lauren Levi ${ }^{1,3}$, Mariela Padilla $^{4}$, Reyes Enciso ${ }^{5}$ \\ ${ }^{1}$ Master of Science Program in Orofacial Pain and Oral Medicine, Herman Ostrow School of Dentistry of USC, Los Angeles, California, \\ USA \\ ${ }^{2}$ Post-graduate Orthodontic Department, European University College, Dubai, United Arab Emirates \\ ${ }^{3}$ New York University School of Dentistry, New York, New York, USA \\ ${ }^{4}$ Assistant Director of Online Programs, Herman Ostrow School of Dentistry of USC, Los Angeles, California, USA \\ ${ }^{5}$ Division of Dental Public Health and Pediatric Dentistry, Herman Ostrow School of Dentistry of University of Southern California, \\ Los Angeles, California, USA
}

\begin{abstract}
This systematic review focused on the efficacy of topical products in reducing temporomandibular joint disorder (TMD)-associated pain, in comparison to placebo or control interventions. The EMBASE, Web of Science, Cochrane Library, and MEDLINE via PubMed databases were searched for randomized controlled trials (RCTs) using topical interventions in adults diagnosed with TMD. The pain intensity was the primary outcome, and other clinical findings were the secondary outcomes. The risk of bias was evaluated according to the Cochrane's handbook. The search up to February 7, 2020 identified a total of 496 unduplicated references. Nine RCTs with 355 adult patients diagnosed with TMD were included. The meta-analysis did not show a significant reduction in baseline pain intensity in the nonsteroidal anti-inflammatory drug (NSAIDs) group, when compared to the placebo group $(\mathrm{P}=0.288)$. One study demonstrated a statistically significant pain score decrease for Theraflex-TMJ compared to placebo after $10 \mathrm{~d}$ of treatment $(\mathrm{P}=0.003)$ and follow-up, $5 \mathrm{~d}$ after the last application $(\mathrm{P}=$ 0.027). Ping On reduced pain at 4 weeks of application $(\mathrm{P}<0.001)$ but not after $7 \mathrm{~d}$ of application $(\mathrm{P}=$ 0.136). In one study, cannabidiol (CBD) significantly improved the pain intensity compared to placebo $(\mathrm{P}<$ 0.001). However, no differences were found with capsaicin in the two studies $(\mathrm{P}=0.465)$. Evidence was of low quality because the studies were considered as having an unclear or a high risk of bias and a small number of studies were analyzed. The evidence is not sufficient to support the use of topical NSAIDs and capsaicin, and limited evidence was found for Threraflex-TMJ, bee venom, Ping On, and CBD, with only one study reporting for each. Additional studies are recommended to validate these results.
\end{abstract}

Keywords: Meta-analysis; Systematic Review; Temporomandibular Joint Disorders; Topical Administration; Visual Analog Scale.

This is an Open Access article distributed under the terms of the Creative Commons Attribution Non-Commercial License (http://creativecommons.org/licenses/by-nc/4.0/) which permits unrestricted non-commercial use, distribution, and reproduction in any medium, provided the original work is properly cited.

\section{INTRODUCTION}

Temporomandibular disorders (TMDs) comprise a collection of conditions in the masticatory muscles, temporomandibular joint (TMJ), and other related structures [1-4]. TMD-related pain could be classified as acute or chronic, and patients may experience restrictions

Received: August 27, 2020 - Revised: October 23, 2020 - Accepted: November 23, 2020

Corresponding Author: Reyes Enciso, Division of Dental Public Health and Pediatric Dentistry, Herman Ostrow School of Dentistry of University of Southern California, 925 West 34th Street, Room \#4268, Los Angeles, CA 90089, USA

Tel: (213)821-6730 Fax: (213) 740-8815 E-mail: renciso@usc.edu

Copyright(C) 2020 Journal of Dental Anesthesia and Pain Medicine 
of jaw movements $[5,6]$. The pain may radiate to the head and neck region and produce other symptoms, such as tinnitus and earaches [3]. Furthermore, the progression, treatment outcome, and occurrence of TMD may be affected by behavioral and psychological components [7].

The "Diagnostic Criteria for Temporomandibular Disorders (DC/TMD) for Clinical and Research Classifications" divides TMDs into joint and muscle problems and then sets up five joint-and six musclerelated subcategories [8]. The International Classification of Orofacial Pain is a recent classification system that incorporates DC/TMD and classifications established by the International Association for the Study of Pain [9].

The first-line treatment for most TMD cases consists of conservative therapy to address the etiologic and risk factors [7]. This often includes dietary modifications, occlusal splint fabrication, physical therapy, behavioral modifications to change a patient's parafunctional habits, patient education, systemic anti-inflammatory drugs, and analgesic medications $[4-7,10]$. The extended use of nonsteroidal anti-inflammatory drugs (NSAIDs) has been associated with significant adverse effects, such as gastrointestinal, renal, hepatic, and cardiovascular events [11-14]. In patients who cannot tolerate these side effects, topical medications may be a therapeutic alternative [15]. Topical formulations applied to the affected area may deliver effective doses at the local site, minimizing the adverse effects associated with systemic doses [16].

Topical NSAIDs (e.g. diclofenac sodium and methyl salicylate) and nutraceuticals (e.g. capsaicin, bee venom, and cannabidiols [CBDs]) have been documented as options in the management of TMD [2,17-24]. Topical interventions could provide patients with TMD with a better safety profile and a noninvasive option to reduce their pain. A systematic review in 2012 evaluated topical nonsteroidal medications, but it did not include nutraceuticals [15]. Thus, the present systematic review aimed to assess the efficacy of topical interventions in reducing pain and other secondary outcomes associated with TMD.

\section{METHODS}

\section{Research question}

This systematic review follows the Preferred Reporting Items for Systematic Reviews and Meta-Analyses guidelines [25] with the protocol registered in PROSPERO (\#CRD42020160723). The PICOS question was as follows: Are topical interventions effective in reducing pain in patients with TMD?

- Population: patients with TMD

- Intervention: topical interventions

- Comparison: placebo or control therapy

- Outcome: reduction in pain (primary) and secondary outcomes, including mouth opening $(\mathrm{mm})$, pain pressure threshold (PPT, pressure applied with a calibrated algometer), TMJ tenderness, and muscle tonus

- Setting: hospital or community-based facility or academic clinical care center

\section{Inclusion and exclusion criteria}

Randomized controlled clinical trials (RCTs) documenting the efficacy of topical interventions in reducing TMD-associated pain and comparing the efficacy with those of placebo or control therapy were included. Clinical guidelines, literature reviews, and systematic reviews were scanned for relevant RCTs. Articles not available in English were not included.

\section{Search methods for study identification}

The search was conducted in four four electronic databases (EMBASE, the Web of Science, Cochrane Library, and MEDLINE via PubMed) up to January 24, 2019 using the strategies described in Table 1 . The search was repeated on February 7, 2020 in the four previously indicated databases, and no additional relevant references were found.

\section{Data collection and analysis}

All the references found using the previously described strategy were assessed by three authors. Duplicates were 
excluded. The titles and abstracts of all records were evaluated based on the inclusion and exclusion criteria. The full articles were then reviewed if a consensus was not met. If a disagreement among the three reviewers still existed after reviewing the entire article, the fourth author decided the final inclusion. Three authors scanned all systematic reviews, clinical guidelines, and reviews from the initial search as well as all eligible RCTs to identify additional relevant references. Any applicable study, which was not included in the initial search, was evaluated using the same criteria and assessed by the same group of authors. If any disagreement arose, they reviewed the full text, and a fourth author made the final decision.

\section{Data extraction and management}

Three authors independently extracted data from the full-text articles of selected and eligible studies with a standardized data entry form. The extracted data included study design, funding source, type of study, country, intervention and sample size, control intervention and sample size, sex, age, co-interventions, adverse events, and outcomes. A fourth author resolved disagreements based on the information and data extracted from the three authors.

\section{Risk of bias assessment in included studies}

The risk of bias per study was assessed by three reviewers and then reviewed by a fourth author, according to the approach described in the Cochrane Handbook [26].

\section{Statistical analyses}

Considering the heterogeneity of the interventions (NSAIDs, nutraceuticals), individual meta-analyses were performed for topical NSAIDs versus placebo/control, Theraflex-TMJ versus placebo, topical NSAIDs versus oral NSAIDs, and each nutraceutical (capsaicin, CBD, Ping On) versus placebo. Treatment effects were recorded as differences in means (DMs) in the change from baseline in pain intensity (0-100 visual analog scale
[VAS]), with confidence intervals (CIs) of 95\%. Statistical heterogeneity was evaluated using the Cochran Q test [27] and I2 statistic [28]. A random-effects model was used to calculate effect estimates if heterogeneity was found ( $\mathrm{Q}$ test, $\mathrm{P}<0.10$ ); otherwise, the fixed-effects model was used. Statistical analyses were performed using the Comprehensive Meta-analysis software version 3 (Biostat, Englewood, NJ, USA). Due to the small number of studies analyzed, the sensitivity analyses for studies with a low risk of bias could not be conducted; a funnel plot to assess for publication bias could not be created as well.

\section{Quality of evidence}

Evidence was assessed with GRADE profiler (GRADEpro) software, following the guidelines of the Cochrane Collaboration and GRADE Working Group [26].

\section{RESULTS}

\section{Results of the search}

Four electronic databases were initially searched up to January 24, 2019 using the strategies described in Table 1. The initial search provided 554 references, and two additional papers were identified after scanning the reference section of the included studies. After removing duplicate papers, 496 references were scanned independently by three authors. Based on the titles and abstracts, only 16 relevant articles were included and their full-text was examined and independently analyzed by the same 3 review authors for inclusion. Seven manuscripts were relevant and included in the analysis. The following articles were excluded: not an RCT $(\mathrm{n}=1)$, proceeding or abstract $(\mathrm{n}=1)$, not in English language $(\mathrm{n}=1)$, different conditions (no TMD, $\mathrm{n}=5$ ), or editorial ( $\mathrm{n}=$ 1). The search was repeated in all four databases on February 7, 2020 and two [17,24] new relevant references were found; thus, a total of 9 studies were included in the analysis (Fig. 1). 
Table 1. Electronic database search strategies

Electronic database

Search strategy

MEDLINE via PubMed search ("Temporomandibular Joint Disorders"[Mesh] OR "Temporomandibular Joint Dysfunction Syndrome"[Mesh] OR "TMD" OR strategy: "TMJ pain" OR "TMJ arthritis" OR "TMJ" OR "TMJ capsulitis" OR "TMJ arthralgia" OR "Myofascial pain" OR "Orofacial (searched up to 1/24/2019; Pain" OR "Facial Pain"[Mesh]) AND ("Administration, Topical/therapy"[Mesh] OR "topical" OR cream OR patch) AND re-run on $2 / 7 / 2020)$ ("Capsaicin"[Mesh] OR "Bee Venom"[Mesh] OR "Anti-Inflammatory Agents, Non-Steroidal"[Mesh] OR "Bee venom" OR "Ping " OR "NSAIDS" OR "Non-Steroidal anti-inflammatory" OR "nonsteroidal anti-inflammatory" OR diclofenac OR "Theraflex" OR " muscle relaxant")

LanguageEnglish

Species: humans

Article types: randomized controlled trials, clinical trails, systematic reviews, review, meta-analysis, guideline, practice guideline

The Web of Science and (Icapsaicin OR "Bee venom" OR "Ping " OR "Non-Steroidal anti-inflammatory" OR "nonsteroidal anti-inflammatory" OR The Cochrane Library diclofenac OR "Theraflex" OR (muscle relaxant)) AND ("TMD" OR (TMJ pain) OR (TMJ arthritis) OR "TMJ" OR (TMJ

(searched up to 1/24/2019; capsulitis) OR (TMJ arthralgia) OR (Myofascial pain) OR (Orofacial Pain) OR (facial pain))) AND ("topical" OR cream re-run on $2 / 7 / 2020)$ OR patch)

EMBASE (searched up to 1/24/2019; re-run on $2 / 7 / 2020$ \#1 'capsaicin'/exp OR capsaicin OR 'bee venom'/exp OR 'bee venom' OR OR 'non-steroidal anti-inflammatory' OR 'nonsteroidal anti-inflammatory' OR 'diclofenac'/exp OR diclofenac OR 'theraflex' OR (('muscle'/exp OR muscle) AND relaxant) \#2 'topical' OR cream OR patch \#3 'tmd' OR (tmj AND pain) OR (tmj AND arthritis) OR 'tmj' OR (tmj AND capsulitis) OR (tmj AND arthralgia) OR (myofascial AND pain)

\#4. \#1 and \#2 and \#3
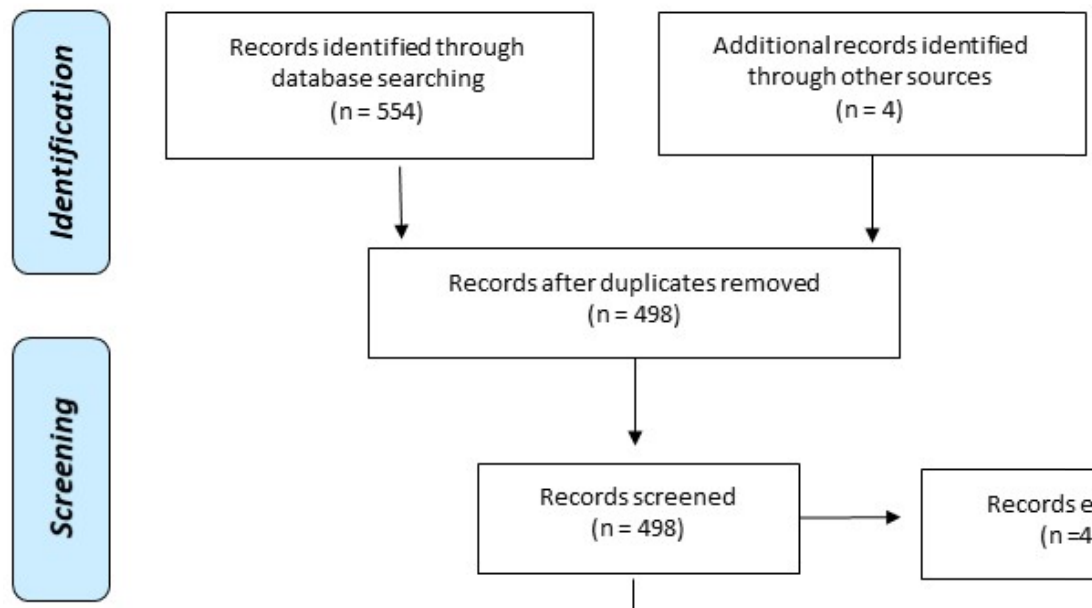

Records after duplicates removed $(n=498)$
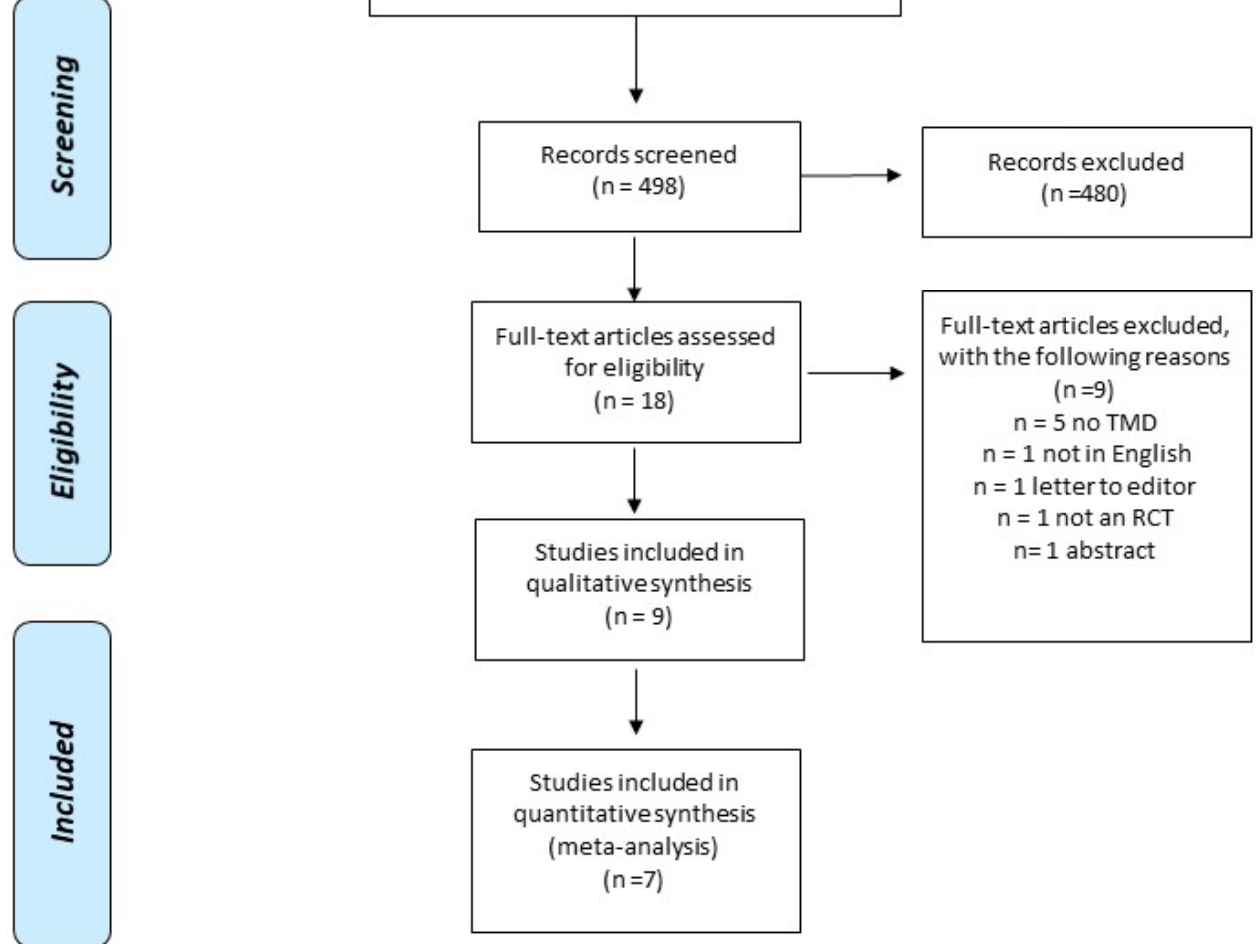

Fig. 1. Preferred Reporting Items for Systematic Reviews and Meta-Analyses (PRISMA) flow diagram [25]. 
Table 2. Summary of eligible RCT studies

\begin{tabular}{|c|c|c|c|c|c|}
\hline Reference & $\begin{array}{l}\text { Country, } \\
\text { dtudy type }\end{array}$ & $\begin{array}{l}\text { Interventions and sample } \\
\text { size per group }\end{array}$ & Temporomandibular disorder diagnosis & Sex & $\begin{array}{l}\text { Age (mean } \pm \\
\text { standard deviation) } \\
\text { [range], yr }\end{array}$ \\
\hline $\begin{array}{l}\text { Campbell } \\
\text { et al. [17] }\end{array}$ & $\begin{array}{l}\text { USA } \\
\text { DBRCT } \\
\mathrm{N}=16\end{array}$ & $\begin{array}{l}\text { I: } 8 \% \text { Capsaicincapsaicin, } \mathrm{n} \\
=8 \\
\text { C: vehicle cream, } \mathrm{n}=8\end{array}$ & $\begin{array}{l}\text { 1. RDC/TMD group Illa criteria for arthralgia of the TMJ } \\
\text { (Dworkin and LeResche [61]); } 2 \text {. TMJ pain of greater than } \\
6 \text { month duration; } 3 \text {. VAS pain score } \geq 3 \text { out of } 10 \text { for } \\
\text { the previous week or the day of testing; } 4 \text {. Subjects had } \\
\text { to have a joint component with or without muscle pain } \\
\text { but were excluded if they only had myalgia. }\end{array}$ & $0 \mathrm{M} / 16 \mathrm{~F}$ & $27.4 \pm 11.9$ \\
\hline $\begin{array}{l}\text { Di Rienzo } \\
\text { Businco } \\
\text { et al. [18] }\end{array}$ & $\begin{array}{l}\text { Italy } \\
\text { Not blinded } \\
\text { RCT } \\
\mathrm{N}=36\end{array}$ & $\begin{array}{l}\text { I: Topical topical diclofenac, } \\
n=18 \\
\text { C: Oral oral diclofenac, } n= \\
18\end{array}$ & $\begin{array}{l}\text { Dysfunction of the } \\
\text { temporomandibular joint - details on of diagnosis diagnostic } \\
\text { criteria not specified. }\end{array}$ & $\begin{array}{l}19 F / 17 \\
M\end{array}$ & $\begin{array}{l}M= \\
43 \mathrm{yr} \\
{[34-61 \text { years }]}\end{array}$ \\
\hline Li et al. [2] & $\begin{array}{l}\text { Hong Kong } \\
\text { DBRCT } \\
\mathrm{N}=45\end{array}$ & $\begin{array}{l}\text { I: Ping On } \\
\text { Ointmentointment, } n=28 \\
\text { C: Petroleum petroleum } \\
\text { jelly, } n=27\end{array}$ & $\begin{array}{l}\text { 1. RDC/TMD Group I criteria [61]; } 2 \text {. For joint and muscle } \\
\text { pain complaints, subjects were required to self-report of } \\
\text { pain occurring daily or nearly for at least } 1 \text { month of daily } \\
\text { or nearly daily pain; } 3 \text {. Subjects subjects with myogenic } \\
\text { pain were included if they met the inclusion and exclusion } \\
\text { criteria }\end{array}$ & $\begin{array}{l}13 \mathrm{M} / 32 \\
\mathrm{~F}\end{array}$ & $\begin{array}{l}\text { I: } 44.0 \pm 13.1 \\
\text { C: } 47.1 \pm 9.3\end{array}$ \\
\hline $\begin{array}{l}\text { Lobo Lobo } \\
\text { et al. [19] }\end{array}$ & $\begin{array}{l}\text { USA } \\
\text { DBRCT } \\
\mathrm{N}=52\end{array}$ & $\begin{array}{l}\text { I: Theraflex-TMJ cream, } n \\
=26 \\
\text { C: Placebo placebo cream, } \\
n=26\end{array}$ & $\begin{array}{l}\text { 1. Pain report in the masseter muscle either at rest or } \\
\text { during when performing functiondaily activities; } 2 \text {. Pain pain } \\
\text { on palpation of the masseter muscle; } 3 \text {. Pain pain in the } \\
\text { temporomandibular joint either at rest or when performing } \\
\text { daily activitiesduring function }\end{array}$ & $5 \mathrm{M} / 47 \mathrm{~F}$ & Age not specified \\
\hline $\begin{array}{l}\text { Nitecka-Buc } \\
\text { hta et al. } \\
{[20]}\end{array}$ & $\begin{array}{l}\text { Poland DBRCT } \\
\mathrm{N}=68\end{array}$ & $\begin{array}{l}\text { I: Bee bee venom ointment, } \\
\mathrm{n}=37 \\
\text { C: Vaseline, } \mathrm{n}=42\end{array}$ & Polish version of RDC/TMD Group la and Ib [62] & $\begin{array}{l}10 \mathrm{M} / 58 \\
\mathrm{~F}\end{array}$ & $\begin{array}{l}\text { mean Mean }=23 \\
y r \\
{[22-34 \quad y r]}\end{array}$ \\
\hline $\begin{array}{l}\text { Nitecka-Buc } \\
\text { hta et al. } \\
{[24]}\end{array}$ & $\begin{array}{l}\text { Poland DBRCT } \\
\mathrm{N}=60\end{array}$ & $\begin{array}{l}\text { I: CBD formulation, } n=30 \\
\text { C: control formulation, } n= \\
30\end{array}$ & Polish version of RDC/TMD Group la and Ib [62] & $\begin{array}{l}\text { 27M/33 } \\
F\end{array}$ & $\begin{array}{l}\text { I: } 23.2 \pm 1.6 \mathrm{yr} \\
\text { C: } 22.6 \pm 1.9 \mathrm{yr}\end{array}$ \\
\hline $\begin{array}{l}\text { Shin \& and } \\
\text { Choi [22] }\end{array}$ & $\begin{array}{l}\text { Korea } \\
\text { DBRCT } \\
\mathrm{N}=20\end{array}$ & $\begin{array}{l}\text { I: } 1 \% \text { indomethacin cream, } \\
\mathrm{n}=10 \\
\mathrm{C}: \text { Ultrasound ultrasound } \\
\text { gel, } \mathrm{n}=10\end{array}$ & $\begin{array}{l}\text { Chief complaint of Temporomandibular temporomandibular } \\
\text { joint pain and tenderness on upon palpation of } \\
\text { temporomandibular joint. }\end{array}$ & $5 \mathrm{M} / 15 \mathrm{~F}$ & $\begin{array}{l}\text { I: } 26.2 \pm 13.5 \mathrm{yr} \\
\text { C: } 18.7 \pm 4.6 \mathrm{yr}\end{array}$ \\
\hline $\begin{array}{l}\text { Wilson F. } \\
{[23]}\end{array}$ & $\begin{array}{l}\text { Canada } \\
\text { DBRCT } \\
\mathrm{N}=28\end{array}$ & $\begin{array}{l}\text { I: Pennsaid, } \mathrm{n}=14 \\
\text { C: } 45.5 \% \text { dimethyl sulfoxide } \\
\text { [DMSO]solution, } \mathrm{n}=14\end{array}$ & $\begin{array}{l}\text { 1. Primary degenerative joint disease as diagnosed through } \\
\text { by radiographs radiography utilizing Conecone-Beam beam } \\
\text { CT; } 2 \text {. A a baseline score } \geq 30 \mathrm{~mm} \text { on a 100- mm VAS } \\
\text { scale (moderate pain) [63]. }\end{array}$ & $0 \mathrm{M} / 28 \mathrm{~F}$ & $\begin{array}{l}\text { I: } M=30.4 \mathrm{yr} \\
{[19-44 \mathrm{yr}]} \\
C: M=28.4 \mathrm{yr} \\
{[18-44 \mathrm{yr}]}\end{array}$ \\
\hline $\begin{array}{l}\text { Winocur } \\
\text { et al. [21] }\end{array}$ & $\begin{array}{l}\text { Israel } \\
\text { DBRCT } \\
\mathrm{N}=30\end{array}$ & $\begin{array}{l}\text { I: active capsaicin cream, } \\
\mathrm{n}=17 \\
\mathrm{C}: \text { Placebo placebo cream, } \\
\mathrm{n}=13\end{array}$ & $\begin{array}{l}\text { 1. History of TMJ pain for at least } 3 \text { months in a defined } \\
\text { area; } 2 \text {. Pain pain in the joint area associated with function; } \\
\text { 3. Presence presence of TMJ tenderness to upon palpation } \\
\text { on theof the reported affected side. }\end{array}$ & $6 \mathrm{M} / 24 \mathrm{~F}$ & $\begin{array}{l}\text { I: } 35.6 \pm 14.2 \\
\text { C: } 37.5 \pm 16.7\end{array}$ \\
\hline
\end{tabular}

Legend: DBRCT, double-blinded randomized controlled trial; RDC/TMD, Research Diagnostic Criteria for Temporomandibular Disorders; I, intervention; C, control; $n$, subject number; M, male; F, female; VAS, visual analog scale; TMJ, temporomandibular joint; TMD, temporomandibular disorders; DMSO, dimethyl sulfoxide; CBD, Cannabidiol; $\mathrm{m}$, mean; $\mathrm{M}$, median.

\section{Included studies}

Nine studies were qualified for the qualitative analysis of the efficacy of topical medications in the treatment of TMD [2,17-24] (Table 2). The studies included in this review were eight randomized, double-blinded placebo-controlled clinical trials and one not blinded placebo-controlled RCT [18].

\section{1) Population}

The total patient sample size included in this review was 355 patients (272 females, 83 males). Patients' age ranged from 18 to 61 years, although not all studies reported the patients' age range. These studies were conducted in Europe [18,20,24], North America [17,19, 23], Asia [2,22], and Middle East [21] (Table 2).

The inclusion criteria were as follows: a) TMD 
Table 3. Details of the interventions

\begin{tabular}{|c|c|c|c|c|c|c|}
\hline \multirow[t]{2}{*}{ Reference } & \multicolumn{3}{|c|}{ Topical intervention } & \multicolumn{2}{|c|}{ Control therapy } & \multirow{2}{*}{$\begin{array}{c}\mathrm{Tx} \\
\text { duration } \\
\end{array}$} \\
\hline & $\begin{array}{l}\text { Intervention's main } \\
\text { component(s), } \\
\text { manufacturer }\end{array}$ & Other ingredients & $\begin{array}{l}\text { Administration } \\
\text { route, dosage, and } \\
\text { instructions }\end{array}$ & Main ingredients & $\begin{array}{l}\text { Route, dose, } \\
\text { and } \\
\text { instructions }\end{array}$ & \\
\hline $\begin{array}{l}\text { Campbell } \\
\text { et al. [17] }\end{array}$ & $\begin{array}{l}\text { Capsaicin 8\% cream } \\
\text { (Westlab Pharmacy, } \\
\text { Gainesville, FL, USA) }\end{array}$ & $\begin{array}{l}\text { Andydrous ointment, butylated } \\
\text { hydroxytoleune NF, polysorbate } 80 \text {, } \\
\text { water }\end{array}$ & Topical, $0.1 \mathrm{~mL}$ & $\begin{array}{l}\text { Vehicle cream } \\
\text { (andydrous ointment, } \\
\text { butylated hydroxytoleune } \\
\text { NF, polysorbate 80, } \\
\text { water) }\end{array}$ & $\begin{array}{l}\text { Same as the } \\
\text { intervention }\end{array}$ & $\begin{array}{l}1 / \text { xdayd } \\
7 \text { daysd }\end{array}$ \\
\hline $\begin{array}{l}\text { Di Rienzo } \\
\text { Businco } \\
\text { et al. [18] }\end{array}$ & $\begin{array}{l}\text { Diclofenac sodium } \\
\text { solution }\end{array}$ & $\begin{array}{l}\text { In a patented carrier with dimethyl } \\
\text { sulfoxide [DMSO] }\end{array}$ & $\begin{array}{l}\text { Oral, } 16 \mathrm{mg} / \mathrm{ml}, 10 \\
\text { drops }\end{array}$ & Oral diclofenac sodium & $\begin{array}{l}\text { Oral, } 50 \mathrm{mg} \\
\text { twice a day }\end{array}$ & $\begin{array}{l}\text { 4/xdday } \\
14 \text { daysd }\end{array}$ \\
\hline Li et al. [2] & $\begin{array}{l}\text { Ping On Ointment } \\
\text { ointment (18\% } \\
\text { peppermint oil, 20\% } \\
\text { menthol, (Ping On } \\
\text { Ointment Company } \\
\text { Limited, Hong Kong) }\end{array}$ & $\begin{array}{l}8 \% \text { bee wax, } 6 \% \text { birch oil, } \\
6 \% \text { natural camphor, } \\
4 \% \text { eucalyptus oil, } 3 \% \text { aromatic oil } \\
\text { and } 1 \% \text { sandalwood oil) }\end{array}$ & $\begin{array}{l}\text { Topical, rub } \\
\text { ointment }\end{array}$ & $\begin{array}{l}0.5 \% \text { ping Ping On } \\
\text { ointment and petroleum } \\
\text { jelly }\end{array}$ & $\begin{array}{l}\text { Same as the } \\
\text { intervention }\end{array}$ & $\begin{array}{l}5 \text { min. } \\
2 x / \text { dayd } \\
4 \text { weeks }\end{array}$ \\
\hline $\begin{array}{l}\text { Lobo Lobo } \\
\text { et al. [19] }\end{array}$ & $\begin{array}{l}\text { Theraflex-TMJ cream } \\
\text { (active ingredient: } \\
\text { Methyl methyl salicylate) } \\
\text { (NaBob/Rx, San Mateo, } \\
\text { CA) }\end{array}$ & $\begin{array}{l}\text { Copper, Zinc zinc, and } \\
\text { Managanese manganese Amino } \\
\text { amino acid complex, } \\
\text { Lysinelysine-aspartate (dipeptide), } \\
\text { MSM, Aloe aloe vera, highly } \\
\text { purified licorice extract, sweet } \\
\text { almond oil, proprietary herbal } \\
\text { blend, capric/ caprylic triglycerides, } \\
\text { emulsifying agents, and water }\end{array}$ & $\begin{array}{l}\text { Topical, apply } 1 / 4- \\
1 / 2 \text { tsp of cream on } \\
\text { painful muscle or } \\
\text { over painful joint }\end{array}$ & Placebo cream & $\begin{array}{l}\text { Same as the } \\
\text { intervention }\end{array}$ & $\begin{array}{l}2 x / \text { dayd } \\
2 \text { weeks }\end{array}$ \\
\hline $\begin{array}{l}\text { Nitecka-Buc } \\
\text { hta et al. } \\
{[20]}\end{array}$ & $\begin{array}{l}0.0005 \% \text { bee venom } \\
\text { ointment }\end{array}$ & & $\begin{array}{l}\text { Topical, massage } \\
\text { over masseter } \\
\text { muscles }\end{array}$ & Vaseline & $\begin{array}{l}\text { Same as the } \\
\text { intervention }\end{array}$ & $\begin{array}{l}3 \mathrm{x} / \text { dayd } \\
14 \text { daysd }\end{array}$ \\
\hline $\begin{array}{l}\text { Nitecka-Buc } \\
\text { hta et al. } \\
{[24]}\end{array}$ & $\begin{array}{l}\text { Cannabidiol } 2.0 \mathrm{~g} \\
\text { ointment ( } 20 \% \text { CBD oil) }\end{array}$ & $\begin{array}{l}\text { Purified water, } 3.0 \mathrm{~g} \\
\text { Ointment Cholesterol cholesterol, } \\
5.0 \mathrm{~g}\end{array}$ & $\begin{array}{l}\text { Topical, massage } \\
\text { over masseter } \\
\text { muscles }\end{array}$ & $\begin{array}{l}\text { Purified water, } 3.0 \mathrm{~g} \\
\text { Ointment Cholesterol, } \\
5.0 \mathrm{~g}\end{array}$ & $\begin{array}{l}\text { Same as the } \\
\text { intervention }\end{array}$ & $\begin{array}{l}2 \mathrm{x} / \text { dayd } \\
14 \text { daysd }\end{array}$ \\
\hline $\begin{array}{l}\text { Shin \& and } \\
\text { Choi [22] }\end{array}$ & $\begin{array}{l}1 \% \text { indomethacin } \\
\text { (Vigel }^{\circledR} \text {, Chodang } \\
\text { Pharmaceutical Industry } \\
\text { Inc., Korea) }\end{array}$ & $\begin{array}{l}\text { in In a base consisting primarily } \\
\text { of water and lanolin }\end{array}$ & $\begin{array}{l}\text { Topical, } 1 \% \\
\text { indomethacin with } \\
\text { an US massage to } \\
\text { the TMJ }\end{array}$ & $\begin{array}{l}\text { Ultrasound coupling } \\
\text { medium } \\
\text { (Aquasonic } ® \text {, Parker } \\
\text { Laboratories, USA). }\end{array}$ & $\begin{array}{l}\text { Same as the } \\
\text { intervention }\end{array}$ & $\begin{array}{l}15 \text { min } \\
1 \mathrm{x} / \text { dayd } \\
2 \text { daysd }\end{array}$ \\
\hline $\begin{array}{l}\text { Wilson } \mathrm{F} . \\
{[23]}\end{array}$ & $\begin{array}{l}\text { Pennsaid }^{(\mathbb{R}} \\
\text { (1.5\% diclofenac) }\end{array}$ & $\begin{array}{l}\text { In } 45.5 \% \text { dimethyl sulfoxide } \\
\text { [DMSO] solution). }\end{array}$ & Topical, apply drops & $45.5 \%$ DMSO & $\begin{array}{l}\text { Same as the } \\
\text { intervention }\end{array}$ & $\begin{array}{l}4 \mathrm{x} / \text { daysd } \\
90 \text { daysd }\end{array}$ \\
\hline $\begin{array}{l}\text { Winocur } \\
\text { et al. [21] }\end{array}$ & $\begin{array}{l}0.025 \% \text { capsaicin cream } \\
\text { (Zostrix, RAFA } \\
\text { Laboratories Ltd) }\end{array}$ & & $\begin{array}{l}\text { Topical - rub into } \\
\text { the skin }\end{array}$ & $\begin{array}{l}\text { Ingredients not specified } \\
\text { (also prepared by RAFA) }\end{array}$ & $\begin{array}{l}\text { Same as } \\
\text { intervention }\end{array}$ & $\begin{array}{l}20-30 \text { sec. } \\
4 x / \text { dayd } \\
4 \text { weeks }\end{array}$ \\
\hline
\end{tabular}

Legend: TMJ, Temporomandibular Joint; DMSO, dimethyl sulfoxide; CBD, Cannabidiol; US, ultrasound

diagnosis classified using axis I of the Research Diagnostic Criteria for Temporomandibular Disorders (RDC/TMD) criteria $[2,20,24]$; b) clinical findings of pain in the masseter and/or joint and tenderness in the TMJ [19,21,22]; c) primary degenerative joint disease based on the RDC/TMD criteria Group IIIa [17], or diagnosed by radiography [23] or moderate pain and cone-beam computerized tomography [23]; and d) dysfunction of the TMJ [18].

\section{2) Interventions}

The interventions are described in Table 3. The reviewers grouped them into three categories based on the active ingredient (NSAID or not) and the control group (placebo, dimethyl sulfoxide [DMSO], or oral NSAID).

- Topical NSAIDs (1\% indomethacin cream [22], 1.5\% diclofenac with $45.5 \%$ DMSO [23]) versus topical 
Table 4. Co-interventions and adverse events in included RCTs

\begin{tabular}{|c|c|c|c|c|c|}
\hline Reference & $\begin{array}{l}\text { Interventions and sample } \\
\text { size }\end{array}$ & Co-interventions & Reported adverse events & $\begin{array}{l}\text { No. of events in } \\
\text { the intervention } \\
\text { group }\end{array}$ & $\begin{array}{l}\text { No. of events in } \\
\text { controls }\end{array}$ \\
\hline Campbell et al. [17] & $\begin{array}{l}\text { I: } 8 \% \text { Capsaicincapsaicin, } \mathrm{n} \\
=8 ; \text { C: control, } \mathrm{n}=8\end{array}$ & Not Statedstated & No data & No data & No data \\
\hline \multirow{3}{*}{$\begin{array}{l}\text { Di Rienzo Businco } \\
\text { et al. [18] }\end{array}$} & \multirow{3}{*}{$\begin{array}{l}\text { I: Topical diclofenac, } n= \\
18 \\
\text { C: Oral diclofenac, } n=18\end{array}$} & \multirow{3}{*}{ Not stated } & Epigastric burning & 0 & 12 \\
\hline & & & Oppression & 5 & 7 \\
\hline & & & Epigastralgia & 1 & 16 \\
\hline \multirow[t]{3}{*}{ Li et al. [2] } & \multirow{3}{*}{$\begin{array}{l}\text { I: Ping On } \\
\text { Ointmentointment, } n=28 \\
\text { C: Petroleum jelly, } n=27\end{array}$} & \multirow{3}{*}{$\begin{array}{l}\text { Patients were allowed to } \\
\text { continue using their } \\
\text { analgesics }\end{array}$} & Eye irritation & 6 & 0 \\
\hline & & & Burning sensation & 3 & 0 \\
\hline & & & Itchiness & 1 & 1 \\
\hline $\begin{array}{l}\text { Nitecka-Buchta et } \\
\text { al. [20] }\end{array}$ & $\begin{array}{l}\text { I: Bee venom ointment, } n \\
=37 \\
\text { C: Vaseline, } n=42\end{array}$ & $\begin{array}{l}\text { Massage to the masseter } \\
\text { muscles } 3 \mathrm{x} / \text { day } \mathrm{d} \text { for } 2 \\
\text { weeks }\end{array}$ & Allergic reaction to bee venom & 1 & 3 \\
\hline $\begin{array}{l}\text { Nitecka-Buchta et } \\
\text { al. [24] }\end{array}$ & $\begin{array}{l}\text { l: } C B D \text { formulation, } n=30 \\
\text { C: control, } n=30\end{array}$ & Not Stated stated & No data & No data & No data \\
\hline $\begin{array}{l}\text { Shin \& and Choi } \\
\text { [22] }\end{array}$ & $\begin{array}{l}\text { l: } 1 \% \text { indomethacin cream, } \\
n=10 \\
\text { C: gel, } n=10\end{array}$ & $\begin{array}{l}\text { TMJ ultrasound massage } \\
\text { for } 15 \mathrm{~min} \text {. }\end{array}$ & No data & No data & No data \\
\hline \multirow[t]{3}{*}{ Wilson F. [23] } & \multirow{3}{*}{$\begin{array}{l}\text { I: Pennsaid, } \mathrm{n}=14 \\
\mathrm{C}: 45.5 \% \text { DMSO solution, } \\
\mathrm{n}=14\end{array}$} & \multirow{3}{*}{$\begin{array}{l}\text { Patients were allowed to } \\
\text { use extra strength } \\
\text { acetaminophen for } \\
\text { break-through pain }\end{array}$} & $\begin{array}{l}\text { Gastrointestinal reaction } \\
\text { (Nauseanausea) }\end{array}$ & $1(7.1 \%)$ & 0 \\
\hline & & & $\begin{array}{l}\text { Application site on the skin } \mathrm{Rx}^{*} \\
\text { Dry skin } \\
\text { Paresthesia } \\
\text { Peeling } \\
\text { Burning } \\
\text { Itchy }\end{array}$ & $\begin{array}{ll}5 & (35.7 \%) \\
2 & (14.3 \%) \\
2 & (14.3 \%) \\
3 & (21.4 \%) \\
1 & (7.1 \%) \\
\end{array}$ & $\begin{array}{l}0 \\
0 \\
0 \\
1 \\
0 \\
\end{array}$ \\
\hline & & & $\begin{array}{l}\text { Other reactions* } \\
\text { Halitosis } \\
\text { Altered Taste taste } \\
\text { Dizziness } \\
\text { Xerostomia } \\
\text { *Some subjects had one or more } \\
\text { side effects }\end{array}$ & $\begin{array}{ll}4 & (28.6 \%) \\
1 & (7.1 \%) \\
1 & (7.1 \%) \\
0 & \end{array}$ & $\begin{array}{ll}1 & (7.1 \%) \\
3 & (21.4 \%) \\
0 & \\
1 & (7.1 \%)\end{array}$ \\
\hline Winocur et al. [21] & $\begin{array}{l}\text { I: active capsaicin cream, } \\
n=1 ; C \text { : Placebo placebo } \\
\text { cream, } n=13\end{array}$ & Not stated & $\begin{array}{l}\text { Warm sensation, stinging, } \\
\text { burning, or redness. }\end{array}$ & $\begin{array}{l}13 / 15 \text { mild to } \\
\text { moderate } \\
\text { intensity }\end{array}$ & $\begin{array}{l}2 \text { patients } \\
\text { Patients with } \\
\text { mild symptoms }\end{array}$ \\
\hline
\end{tabular}

Legend: I, intervention; C, control; $n$, subject number; TMJ, temporomandibular joint; DMSO, dimethyl sulfoxide; CBD, cannabidiol.

placebo [22] or DMSO [23].

- Topical Threraflex-TMJ cream versus placebo cream [19].

- One study [18] evaluated the difference between topical NSAID $\quad(16-\mathrm{mg} / \mathrm{ml}$ diclofenac sodium solution versus oral NSAID (50-mg tablet of oral diclofenac sodium administered twice a day after a meal for $14 \mathrm{~d}$.)

- Nutraceuticals (capsaicin [17,21], CBD [24], bee venom [20], Ping On [2]) versus placebo.

All interventions of topical treatments were applied directly by the patient, except for the indomethacin cream that was applied only once through ultrasound treatment (phonophoresis) [22]. Patients were instructed to apply the topical treatment once [17], two [2,19,24], three [20], or four times a day $[18,21,23]$. Treatments lasted between 2 and $90 \mathrm{~d}$ (Table 3). 
Table 5. Summary of risk of bias for eligible RCT studies

\begin{tabular}{|c|c|c|c|c|c|c|c|}
\hline Study & $\begin{array}{c}\text { Random } \\
\text { sequence } \\
\text { generation }\end{array}$ & $\begin{array}{c}\text { Allocation } \\
\text { concealment }\end{array}$ & Blinding & $\begin{array}{l}\text { Incomplete } \\
\text { outcome } \\
\text { data }\end{array}$ & Selective reporting & $\begin{array}{c}\text { Other } \\
\text { potential } \\
\text { bias }\end{array}$ & Overall bias \\
\hline Campbell et al. [17] & - & - & $?$ & - & - & $?$ & $?$ \\
\hline Di Rienzo Businco et al. [18] & $?$ & $?$ & $?$ & - & - & $?$ & $?$ \\
\hline Li et al. [2] & - & - & $?$ & - & - & $?$ & $?$ \\
\hline Lobo Lobo et al. [19] & - & - & $?$ & - & - & $?$ & $?$ \\
\hline Nitecka-Buchta et al. [20] & - & - & $?$ & - & - & $?$ & $?$ \\
\hline Nitecka-Buchta et al. [24] & - & $?$ & $?$ & - & - & $?$ & $?$ \\
\hline Shin \& and Choi [22] & $?$ & $?$ & $?$ & $?$ & - & $?$ & $?$ \\
\hline Wilson F. [23] & - & - & $?$ & + & - & $?$ & + \\
\hline Winocur et al. [21] & $?$ & $?$ & $?$ & - & - & $?$ & $?$ \\
\hline
\end{tabular}

KEY: " -" is low; "+" is high and "?" is unclear risk of bias.

\section{3) Comparison groups}

Patients in the comparison groups received a non-active vehicle (petroleum jelly [2], Vaseline [20], placebo cream $[17,19,21,24]$, or inert ultrasound gel [22]) or control therapy, including oral diclofenac [18] or 45.5\% DMSO [23] (Table 3).

\section{4) Co-interventions}

Two studies mentioned that rescue medications were allowed during the study $[2,23]$. One study allowed the patients to continue their analgesic treatment [2], and the other study used extra strength acetaminophen for patients with breakthrough pain [23] (Table 4).

\section{5) Outcomes}

The primary reported outcome was change in joint pain, measured using the $0-10$ VAS [17,18,20,21,24], 0-100 VAS [2,22,23], or 0-10 Numerical Graphic Rating Scale (NGRS) [19]. For VAS 0-10, 0 indicates no pain, whereas 10 indicates worst pain. For 0-100 VAS, 0 indicated no pain and 100 indicates worst pain. In the VAS, the patient draws a line on a $0-10-\mathrm{cm}$ or a $0-100-\mathrm{mm}$ scale. The NGRS is a verbal scale. Secondary outcomes included mouth opening, defined as maximum vertical mouth opening (in millimeters) without evoking pain [2], voluntary and assisted vertical mouth opening [23], and active and passive mouth opening [21]. Other secondary outcomes included joint tenderness (VAS)
$[2,18]$, muscle pain (VAS) [20], functional limitation of mouth opening ( 0 , difficulty in opening mouth; 5, maximum mouth opening) [18], PPT measured with an algometer [22], muscle tenderness (score 0-3) [21], pain disability index scores [23], median values of muscular tonus [20], adverse events, changes in thermal sensitivity (Campbell), and masseter muscle activity as measured by surface electromyography (24).

\section{Risk of bias in included studies}

The risk of bias assessment in nine included studies [2,17-24] is presented in Table 5 and Fig. 2 (graph of risk of bias).

Random Sequence Generation: Six studies [2,17,19, 20,23 ] were defined as having a low risk for random sequence generation (considering randomized envelope selection, block randomization, and a random digit table). Three studies $[18,21,22]$ were assessed to have an unclear risk, since the authors stated that the studies were randomized, but there was no mention on how the randomization was performed.

Allocation concealment: Five of the studies [2,17,19, 20,23 ] were considered as having a low risk, since they used sealed envelopes to conceal the random sequence. Four of the studies $[18,21,22,24]$ did not indicate the allocation concealment method; thus, they were considered as having an unclear risk.

Blinding: Blinding of the researchers, participants, and data analysts or assessors were determined. None of the 


\section{Graph of Risk of Bias}

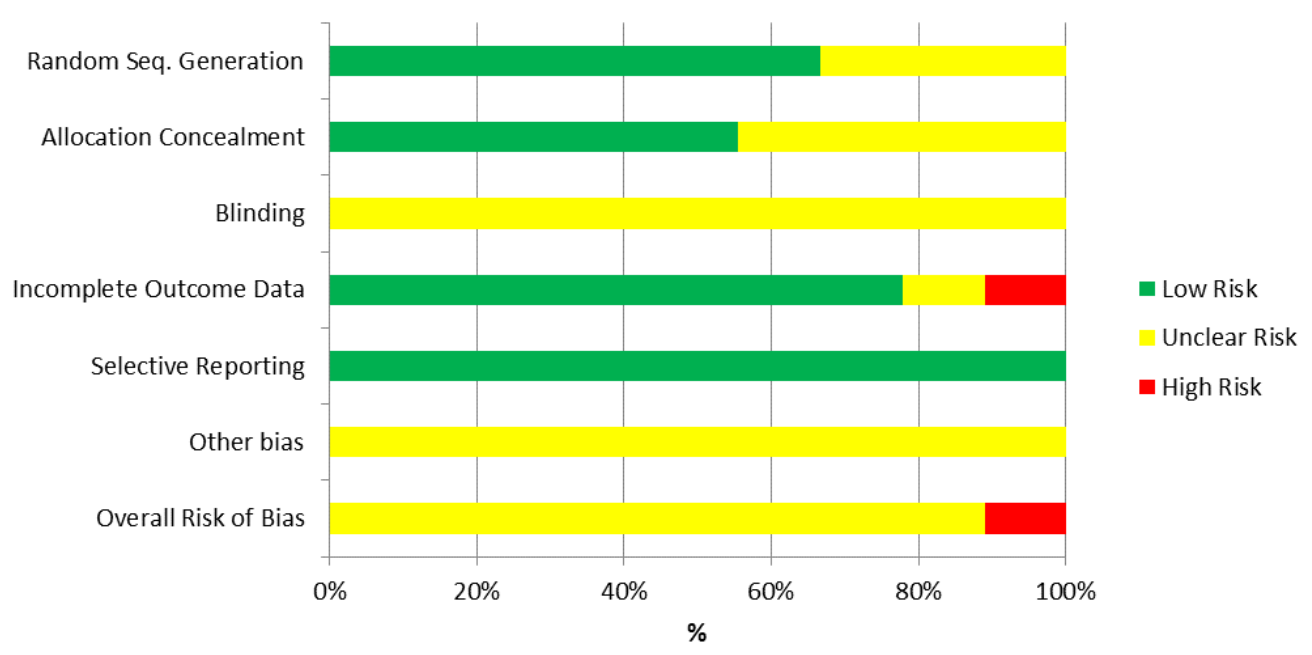

Fig. 2. Summary of risk of bias of eligible randomized controlled trials (RCTs).

studies effectively indicated the blinding process for all participants, investigators, outcome assessors or data analysts in the research; thus, no studies were considered to have a low risk of bias. All nine studies [2,17-24] were considered to have an unclear risk of bias because the authors indicated that their studies were double-blinded; however, there is no indication of the blinding method for all four groups (investigator, data assessors, analyst, participants).

Incomplete Outcome Data: Seven studies [2,17-21,24] did not have missing data; thus, they were considered to have a low risk of bias. One trial [22] was deemed to have an unclear risk because it did not mention any dropouts and confirm if all subjects were reported. One study [23] was at a high risk considering the high number of dropouts (> 20\%).

Selective Reporting: Nine studies [2,17-24] documented the outcomes and were assigned a rating of low risk of bias for selective reporting.

Other Bias: Other forms of bias analyzed were co-interventions, funding sources, and imbalance of baseline groups. Nine studies [2,17-24] were considered at an unclear risk because they did not state any co-interventions or funding, and one study [17] had an imbalance of baseline groups.

Overall Risk of Bias: None of the studies were considered to have a low overall risk for bias. Eight studies [2,17-22,24] were assessed as having an unclear risk, and one study [23] was considered at a high risk for bias due to the large number of dropouts (Table 5; Fig. 2).

\section{Meta-analyses}

Individual studies reporting outcomes are presented in Table 6. Considering the interventions' heterogeneity and comparison groups, the reviewers conducted metaanalyses for change from baseline in VAS pain intensity for two classes of interventions: NSAID versus control groups $(\mathrm{n}=2$; Fig. $3 \mathrm{a})$ and capsaicin versus placebo ( $\mathrm{n}$ $=2$; Fig. $3 b$ ).

Interventions with only one study reporting relevant outcomes (baseline and post-treatment VAS averages and standard seviation [SD]) for Theraflex-TMJ [19] (Fig. 3c), Ping On [2] (Fig. 3d), and CBD [24] (Fig. 3e) are shown for informational purposes only. Two studies did not provide VAS standard deviations of pain intensity for bee venom [20] and topical treatment versus oral diclofenac [18] and could not be analyzed in this meta-analysis.

\section{1) Topical NSAIDS versus control therapy}

In one meta-analysis, this review found a non- 
Table 6. Results reported by included RCTs

\begin{tabular}{|c|c|c|c|c|c|}
\hline Reference & $\begin{array}{l}\text { Intervention vs. } \\
\text { control therapy }\end{array}$ & Outcomes in the intervention group & Outcomes in the control group & $\begin{array}{l}\text { Differences between the } \\
\text { Intervention intervention and } \\
\text { Control control groups(NS: P > } \\
0.05 \text { ) }\end{array}$ & Summary \\
\hline $\begin{array}{l}\text { Campbell et } \\
\text { al. [17] }\end{array}$ & $\begin{array}{l}\text { I: } 8 \% \text { capsaicin } \\
\text { C: vehicle cream }\end{array}$ & $\begin{array}{l}\text { 1. Two hours after application increase } \\
\text { Increase in VAS pain ratings at } 2 \mathrm{~h} \text { after } \\
\text { application. } \\
\text { 2. After } 1 \text { week decrease Decrease in the VAS } \\
\text { pain ratings at } 1 \text { week after application }\end{array}$ & $\begin{array}{l}\text { 1. Short- term effect: less VAS pain ratings } \\
\text { score } \\
\text { 2. Long- term effect: higher VAS pain ratings } \\
\text { score than the Capsaicin capsaicin group }\end{array}$ & $\begin{array}{l}\text { VAS measures during at } 1 \text { to }-7 \\
\text { days } d \text { were } \\
\text { significantly greater in the control } \\
\text { group ( } P=0.001) \text {. However, the } \\
\text { control group initially had started } \\
\text { ata higher VAS pain score at } \\
\text { baseline }\end{array}$ & $\begin{array}{l}\text { Pain } \\
\text { reduction } \\
\text { was similar } \\
\text { in both } \\
\text { groups }\end{array}$ \\
\hline $\begin{array}{l}\text { Di Rienzo } \\
\text { Businco et al. } \\
\text { [18] }\end{array}$ & $\begin{array}{l}\text { I: Topical } \\
\text { diclofenac } \\
\text { C: Oral diclofenac }\end{array}$ & $\begin{array}{l}\text { 1. Relief from pain after treatment ( } p \text {-value } \\
\text { not stated) }\end{array}$ & $\begin{array}{l}\text { 1. relief Relief from pain after treatment } \\
\text { (p-value not stated) }\end{array}$ & $\begin{array}{l}\text { No difference in relief from pain or } \\
\text { mouth opening }\end{array}$ & $\begin{array}{l}\text { No } \\
\text { difference }\end{array}$ \\
\hline Li et al. [2] & $\begin{array}{l}\text { I: Ping On } \\
\text { Ointment } \\
\text { C: Petroleum jelly }\end{array}$ & $\begin{array}{l}\text { VAS pain score decreased significantly }(P< \\
0.001) \\
\text { Maximum Comfortable Mandibular Opening } \\
\text { did increase significantly }(P=0.01)\end{array}$ & $\begin{array}{l}\text { VAS pain score did not decrease } \\
\text { significantly } \\
\text { Maximum Comfortable comfortable } \\
\text { Mandibular mandibular Opening opening } \\
\text { increased but not significantly ( }>0.05 \text { ) }\end{array}$ & $\begin{array}{l}\text { Significant difference between } \\
\text { groups in VAS pain score }(P< \\
0.05) \text { and maximum comfortable } \\
\text { mandibular opening }(P<0.01) \\
\text { between the two groups }\end{array}$ & $\begin{array}{l}\text { Ping On } \\
\text { better than } \\
\text { placebo }\end{array}$ \\
\hline $\begin{array}{l}\text { Lobo Lobo et } \\
\text { al. [19] }\end{array}$ & $\begin{array}{l}\text { I: Theraflex-TMJ } \\
\text { cream } \\
\text { C: placebo cream }\end{array}$ & $\begin{array}{l}\text { Significant decrease in reported pain levels } \\
\text { from baseline after } 10 \text { days } d(P<0.01) \text {, } \\
15 \text { days } d(P<0.001) \text {, and follow-up }(P< \\
0.01) \text {. }\end{array}$ & $\begin{array}{l}\text { For the control group, no significant } \\
\text { differences were found between among the } \\
\text { different time periods }(P>0.05) \text {. }\end{array}$ & $\begin{array}{l}\text { Significant differences in outcomes } \\
\text { between the Theraflex and control } \\
\text { groups at } 15 \text { days } d \text { after tx start } \\
\text { of treatment }(P<0.05) \text {. }\end{array}$ & $\begin{array}{l}\text { Theraflex } \\
\text { was better } \\
\text { than } \\
\text { placebo }\end{array}$ \\
\hline $\begin{array}{l}\text { Nitecka-Bucht } \\
\text { a et al. [20] }\end{array}$ & $\begin{array}{l}\text { I: Bee venom } \\
\text { ointment } \\
\text { C: Vaseline }\end{array}$ & $\begin{array}{l}\text { 1. Changes in VAS scale score before and } \\
\text { after } 2 \text { weeks were statistically significant (P } \\
=0.000002 \text { ). }\end{array}$ & $\begin{array}{l}\text { 1. Changes in VAS scale score before and } \\
\text { after } 2 \text { weeks were statistically relevant } \\
\text { significant ( } P=0.000024) \text {. }\end{array}$ & $\begin{array}{l}\mathrm{P} \text { value of the difference not } \\
\text { reported }\end{array}$ & $\begin{array}{l}\text { Differences } \\
\text { not reported }\end{array}$ \\
\hline $\begin{array}{l}\text { Nitecka-Bucht } \\
\text { a et al. [24] }\end{array}$ & $\begin{array}{l}\text { l: CBD formulation } \\
\text { C: Placebo } \\
\text { formulation }\end{array}$ & $\begin{array}{l}\text { Pain intensity in, measured by VAS, scale } \\
\text { significantly decreased }(70.2 \%)\end{array}$ & $\begin{array}{l}\text { No effect on the average pain level VAS pain } \\
\text { score of on the masseter muscle }\end{array}$ & $\begin{array}{l}\text { Statistically significant reduction in } \\
\text { pain score in CBD group; , but not } \\
\text { significant in the control group }\end{array}$ & $\begin{array}{l}\text { CBD is } \\
\text { better than } \\
\text { Placebo } \\
\text { placebo }\end{array}$ \\
\hline $\begin{array}{l}\text { Shin \& and } \\
\text { Choi [22] }\end{array}$ & $\begin{array}{l}\text { l: } 1 \% \\
\text { indomethacin } \\
\text { cream } \\
\text { C: Placebo cream }\end{array}$ & $\begin{array}{l}\text { 1. Significant improvement in TMJ pain VAS } \\
\text { pain score }(P=0.011) \\
\text { 2. The pressure pain threshold was increased } \\
(P=0.005)\end{array}$ & $\begin{array}{l}\text { No significant improvement in } \\
\text { TMJ-associated Pain pain }(P>0.05) \text { nor } \\
\text { or pressure pain threshold }(P>0.05)\end{array}$ & $\begin{array}{l}\text { Differences between groups not } \\
\text { reported }\end{array}$ & $\begin{array}{l}\text { Differences } \\
\text { not reported }\end{array}$ \\
\hline Wilson F. [23] & $\begin{array}{l}\text { I: Pennsaid (1.5\% } \\
\text { diclofenac) } \\
\text { C: } 45.5 \% \text { DMSO }\end{array}$ & $\begin{array}{l}\text { 1. Functional Pain pain Intensity intensity (100 } \\
\text { mm VAS scale); } P<0.001 \\
\text { 2. No significant difference in voluntary } \\
\text { vertical mouth opening }(P>0.05)\end{array}$ & $\begin{array}{l}\text { 1. Functional Pain pain Intensity }(100 \mathrm{~mm} \\
\text { VAS scale); } P<0.001 \text {; } \\
\text { 2. No difference in vertical mouth opening } \\
(P>0.05)\end{array}$ & $\begin{array}{l}\text { No difference in VAS pain intensity } \\
(P=0.304) \text { nor or in voluntary } \\
\text { vertical mouth opening }(P>0.05)\end{array}$ & $\begin{array}{l}\text { No } \\
\text { difference }\end{array}$ \\
\hline $\begin{array}{l}\text { Winocur et al. } \\
\text { [21] }\end{array}$ & $\begin{array}{l}\text { I: active Active } \\
\text { capsaicin cream } \\
\text { C: Placebo cream }\end{array}$ & $\begin{array}{l}\text { 1. Present Painpain, Maximal maximum Pain } \\
\text { pain intensity, and pain relief scale score } \\
\text { decreased significantly with capsaicin ( } P= \\
0.0001) ; 2 \text {. Active active and Passive passive } \\
\text { Mouth mouth Opening opening increased } \\
\text { significantly }(P<0.05)\end{array}$ & $\begin{array}{l}\text { 1. Present Painpain, Maximal maximal Pain } \\
\text { pain intensity, and pain relief scale score } \\
\text { decreased with placebo }(P=0.0001) ; 2 \text {. } \\
\text { Active active and Passive passive Mouth } \\
\text { mouth Opening opening increased } \\
\text { significantly }(P<0.05)\end{array}$ & $\begin{array}{l}\text { No differences in the pain level on } \\
\text { between either the painful or and } \\
\text { non-painful sides regarding of the } \\
\text { affected muscle and joint } \\
\text { tenderness to palpation. }\end{array}$ & $\begin{array}{l}\text { No } \\
\text { difference }\end{array}$ \\
\hline
\end{tabular}

Legend: I, intervention; C, control; n, subject number; VAS, visual analog scale; TMJ, temporomandibular joint; TMD, temporomandibular disorders; DMSO, dimethyl sulfoxide; CBD, cannabidiol.

significant favorable difference in the improvement of pain intensity (scale 0-100) from baseline in the topical NSAID group when compared to placebo. NSAIDs improved the pain intensity compared to control therapy in two studies $(\mathrm{P}=0.288)$. Pain intensity improved by an average of -6.54 units more from baseline in the NSAID group than in the control group $(95 \% \mathrm{CI}=$ -18.605 to $-5.525 ; \mathrm{P}=0.288$; Fig. 3a).

\section{2) Capsaicin}

In one meta-analysis, capsaicin showed an average improvement in VAS pain score of -4.2 units (0-100 scale) compared to the placebo group in two studies [17, 21] at $7-30 \mathrm{~d}(95 \% \mathrm{CI}=-15.502$ to $7.087 ; \mathrm{P}=0.465$; Fig. 3c). This improvement was not statistically significant. 
3) Theraflex-TMJ versus placebo

Lobo Lobo et al. [19] showed a significant improvement in pain intensity with Theraflex-TMJ cream of -19.5 units (on a 0-100 scale) compared to placebo at day 10 $(95 \% \mathrm{CI}=-19.500$ to $-32.165 ; \mathrm{P}=0.003$; Fig. $3 \mathrm{c})$ and at follow-up $(95 \% \mathrm{CI}=-14.400$ to $-27.192 ; \mathrm{P}=0.027$; Fig. 3c).

A

Change in VAS pain from baseline

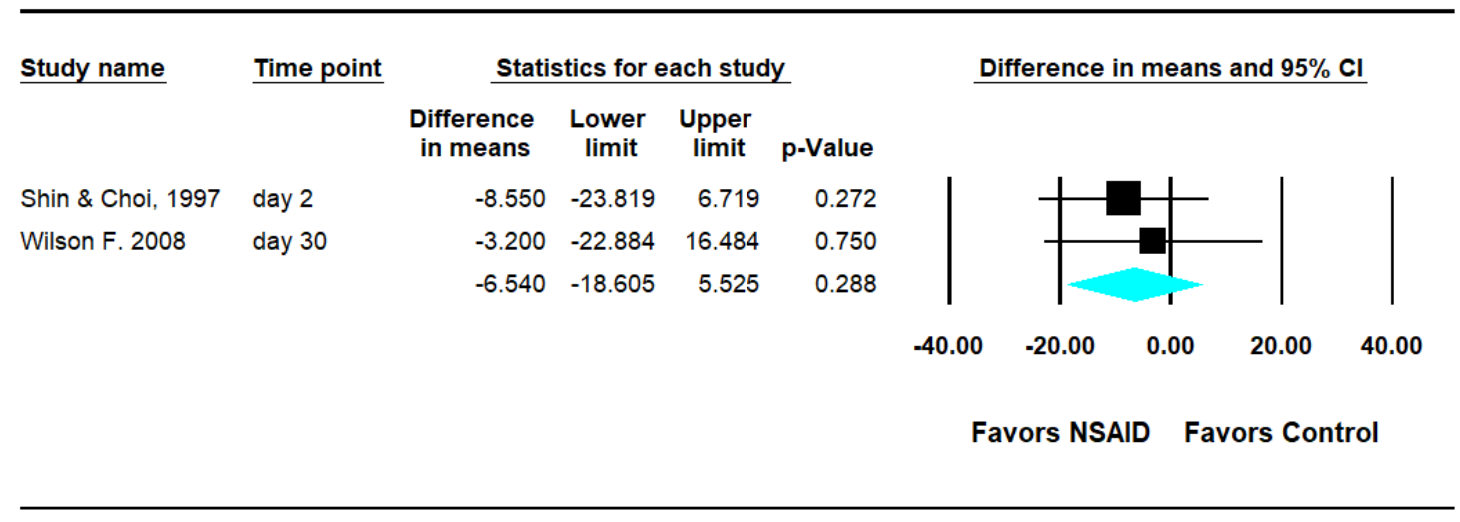

Fixed-effect analysis

B

Change in VAS pain from baseline

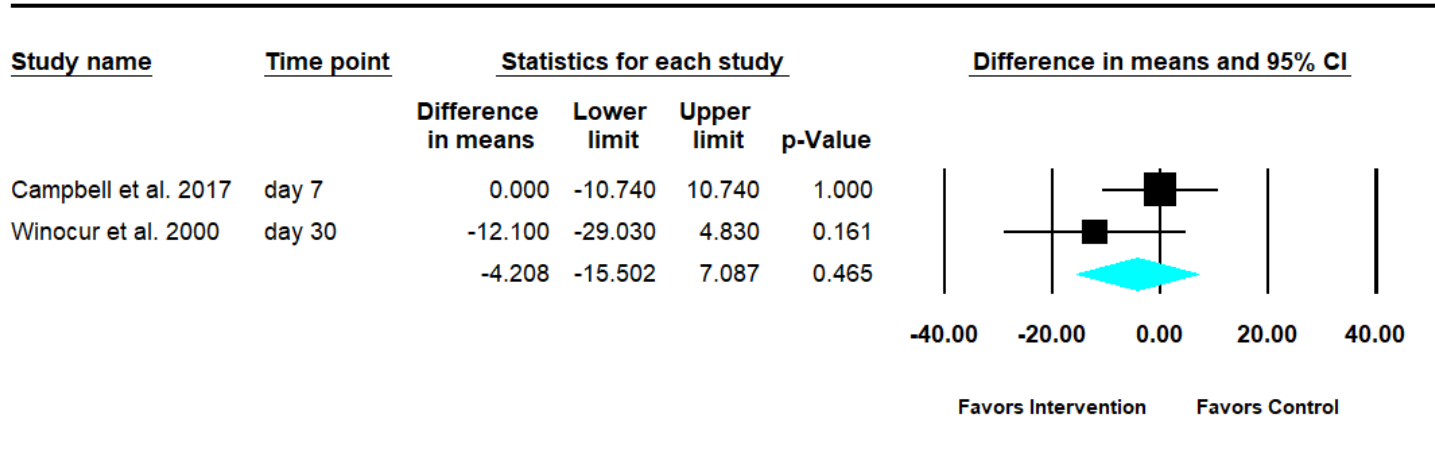

Random-effects analysis

C

\section{Change in VAS pain from baseline}

\begin{tabular}{lllllll} 
Study name & Time point & & \multicolumn{3}{c}{ Statistics for each study } \\
\cline { 2 - 5 } & & $\begin{array}{r}\text { Difference } \\
\text { in means }\end{array}$ & $\begin{array}{c}\text { Lower } \\
\text { limit }\end{array}$ & $\begin{array}{c}\text { Upper } \\
\text { limit }\end{array}$ & p-Value \\
Lobo Lobo et al. 2004 & day 10 & -19.500 & -32.165 & -6.835 & 0.003 \\
Lobo Lobo et al. 2004 & follow up & -14.400 & -27.192 & -1.608 & 0.027
\end{tabular}

Difference in means and $95 \% \mathrm{Cl}$
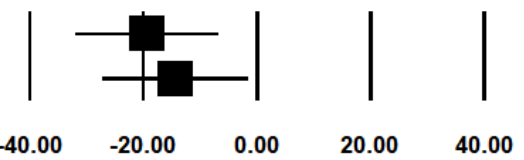

Favors Intervention

Favors Control

Fixed-effect analysis

(continued) 
(continued)

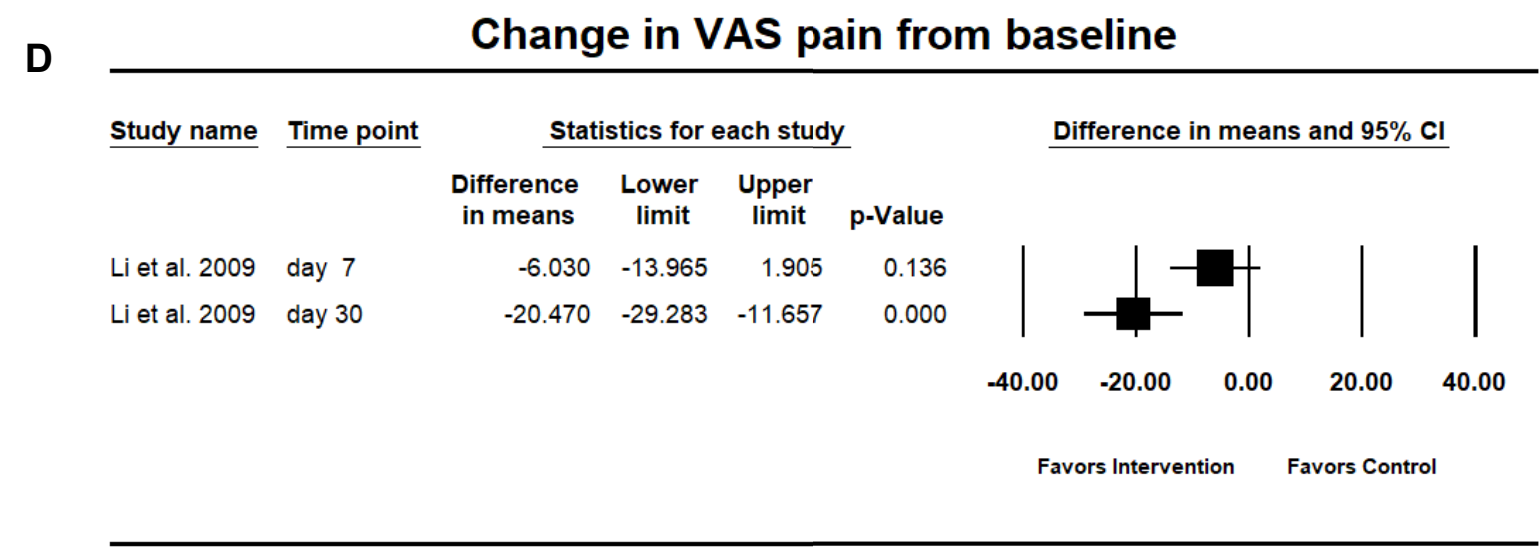

Fixed-effects analysis

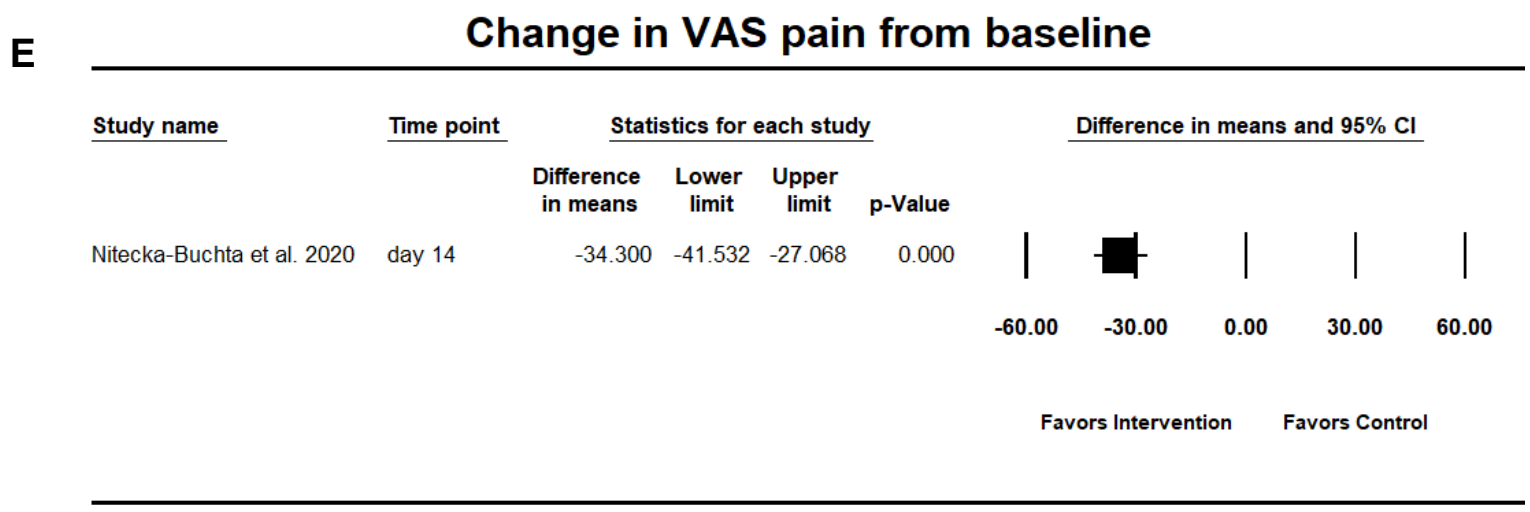

Fixed-effects analysis

Fig. 3. Meta-analyses: nonsteroidal anti-inflammatory drugs (NSAIDs) versus control (a); capsaicin versus control (b); Theraflex-TMJ versus control (c); Ping On versus control (d); and CBD versus control (e).

4) Nutraceuticals versus controls

No meta-analyses could be undertaken for bee venom versus placebo [20], as the authors did not report standard deviations. Thus, we summarize the results for Ping On and CBD with one study for informational purposes. $\mathrm{Li}$ et al. [2] showed a significant improvement in pain intensity with Ping On ointment of -20 units (on a 0-100 scale) compared to placebo at day $30(95 \% \mathrm{CI}=-29.283$ to -29.283 ; $\mathrm{P}<0.001$; Fig. 3d) but not at day 7 (95\% $\mathrm{CI}=-13.965$ to $1.905 ; \mathrm{P}=0.136 ;$ Fig. $3 \mathrm{~d}$ ). Nitecka-Buchta et al. [24] showed a significant improvement in the CBD group in VAS pain score of 34 units compared to placebo in one study at day 14 (95\% $\mathrm{CI}=-41.532$ to $-27.068 ; \mathrm{P}<0.001$; Fig. 3e). Further studies are needed to confirm these findings.

\section{Co-interventions}

Four studies reported to provide co-interventions on the patients $[2,20,22,23]$. In one trial [2], the patients were allowed to continue their analgesic treatment, and, in one RCT [23], patients were allowed to use extra strength acetaminophen for breakthrough pain. In one study [20], patients performed a simple physiotherapy by massaging the masseter muscle area 3 times per day for 2 weeks prior to the follow-up appointment. In another study [22], the treatment method included the use of ultrasound (1.0 $\mathrm{MHz}, 0.8-1.5 \mathrm{~W} / \mathrm{cm}^{2}$ continuous output) over the painful TMJ for 15 minutes (Table 4).

\section{Adverse events}

Adverse events of topical treatments were mainly skin or eye allergy, irritation, redness, or burning sensation 
Table 7. Summary of evidence and quality of the findings (GRADE)

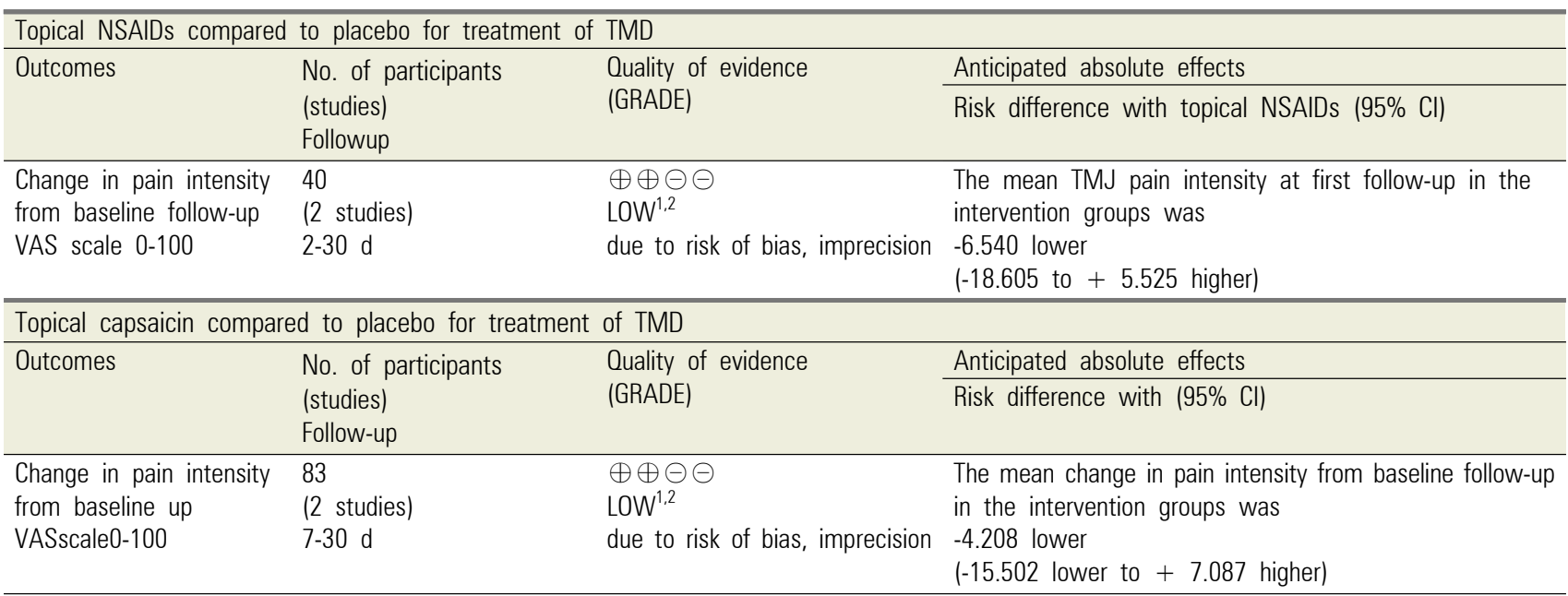

GRADE Working Group grades of evidence

Low quality: Further research is very likely to have an important impact on our confidence in the estimate of effect and is likely to change the estimate.

${ }^{1}$ All studies assessed unclear or high risk of bias

${ }^{2}$ Only two or three studies included in this meta-analysis: small sample size (total number of participants: < 400).

Legend: VAS, visual analog scale; TMJ, temporomandibular joint; TMD, temporomandibular disorders; Cl, confidence intervals.

$[2,18,20,21,23]$, with systemic effects, such as nausea, dizziness, and xerostomia, being rarely reported [21]. Adverse effects of oral diclofenac treatment were mainly epigastric burning or retrosternal oppression sensation [18]. No adverse events were reported by three studies $[17,19,22]$ (Table 4).

\section{Summary of evidence and quality of the findings (GRADE)}

Only the trials with similar outcomes were included in the meta-analyses. The evidence was considered of low quality due to the risk of bias and imprecision (small sample sizes in each meta-analysis $<400$, and a small number of studies per meta-analysis) present for all meta-analyses in this systematic review (Table 7).

\section{DISCUSSION}

\section{Main findings}

\section{1) Topical NSAIDs versus control therapy}

By blocking the cyclooxygenase (COX) pathway and reducing the production of prostaglandins, NSAIDs are commonly used as the first treatment option for TMD $[2,15,19,29]$. Nonetheless, non-selective COX inhibitors are well known to be associated with adverse events, such as increased risk of bleeding and gastrointestinal disturbances [30].

In this review, the topical NSAID (1\% indomethacin cream [22] and Pennsaid [23]) versus control vehicle groups showed a non-significant reduction in the reported pain levels from baseline of -6.54 units on a $0-100$ scale $(\mathrm{P}=0.288)$. Kelly [31] stated that the "minimum clinically significant difference" on a VAS is 12 units (95\% CI $=9-15$ units). The difference in VAS pain score of -6.54 units when comparing topical NSAIDs to placebo groups in this systematic review was not clinically significant.

Different co-interventions might have helped reach the treatment efficacy. Shin and Choi [22] used an ultrasound device to apply the NSAID gel (continuous output of 1.0 $\left.\mathrm{MHz}, 0.8-1.5 \mathrm{~W} / \mathrm{cm}^{2}\right)$. Delivering indomethacin at higher ultrasound frequencies $(\geq 0.7 \mathrm{MHz}$ ) might help in delivering the drug to deeper tissues, since it induces chemical, thermal, or mechanical changes in the cutaneous 
tissue [32,33]. Ramakrishnan et al. [34] investigated the efficacy of phonophoresis in treating TMDs using NSAID gel as a coupling agent versus plain therapeutic continuous ultrasound. The authors found that ultrasound treatment without NSAID gel was effective in reducing pain (VAS) and C-reactive protein (CRP) level, an inflammatory marker, and no statistically significant difference in outcomes were found between patients undergoing treatment with ultrasound and NSAID gel and those undergoing plain therapeutic ultrasound.

In one study [23], the authors used Pennsaid (1.5\% diclofenac in $45.5 \%$ DMSO solution) as the intervention and $45.5 \%$ DMSO as the control; however, the results showed that the control group experienced improvements similar to those experienced by the intervention group. This improvement could be due to the DMSO properties, which include anti-inflammatory, analgesia, nerve blockage, bacteriostatic, and vasodilation [35].

\section{2) Theraflex-TMJ versus placebo}

Theraflex-TMJ (Nabob/Rx, San Mateo, CA), composed of methyl salicylate (derivative of aspirin), which is winter green oil, copper pyrocarboxylate, and zinc pyrocarboxylate, has been investigated as a treatment for masseteric and TMJ-related pain [19]. Copper may provide a therapeutic value in treating arthritis and tissue damage by reducing the production of free radicals and inactivating free radicals produced during inflammation [19]. Prior studies reported that rheumatoid arthritis (RA) patients had higher serum levels of copper and ceruloplasmin than those without arthritis, but not in those with degenerative joint diseases [36,37]. Copper may play a role in taming free radicals, thus reducing inflammation [38]. The mechanism of action of zinc is unclear; however, zinc deficiency is associated with immunosuppression [19,39,40]. Additionally, zinc activates natural killer cells, inhibits inflammatory mediator production, and plays a role in neutrophil function maintenance [41].

The co-interventions might have helped in achieving treatment efficacy. For Theraflex-TMJ, [19] the cream preparation contains not only an NSAID but also copper and zinc. The anti-inflammatory properties of copper [42] and zinc might have improved the results of this study.

\section{3) Topical diclofenac versus oral diclofenac}

With only one study reporting the average pain score changes from baseline, when comparing topical versus oral NSAIDs, and given that no standard deviations were reported, a meta-analysis could not be conducted. Authors of the individual study reported that when comparing post-treatment pain between the topical diclofenac and oral diclofenac groups, the improvement in pain intensity assessment on a 0-10 VAS after treatment was not statistically significant $(\mathrm{P}>0.05)$. Moreover, no significant differences were found in the improvement in the mouth opening between the two groups $(\mathrm{P}>0.05)$. However, the main difference was that patients receiving topical diclofenac had reduced systemic adverse effects, whereas those receiving oral diclofenac showed adverse effects in the gastrointestinal system. The plasma concentration of the topical NSAIDs is small compared to the systemically administered NSAIDs, which in turn reduces the likelihood and risk of renal impairment, cardiovascular events, gastrointestinal adverse reactions, or drug interactions $[16,43,44]$.

It should not be ignored that some cutaneous adverse reactions occur due to topical NSAID applications, such as pruritis, irritation, burning or heat sensation, and contact dermatitis, but they are most likely easy to control and self-limiting [45]. Hsieh et al. [45] found that the control group (menthol patches) had skin irritation and erythema more frequently than the treatment group (diclofenac sodium patch), suggesting that the carrier, not diclofenac, could be the cause.

\section{4) Capsaicin}

By eliciting the release of substance $\mathrm{P}$ and targeting the vanilloid 1 transient receptor potential, capsaicin has been utilized in the treatment of neuralgias, RA, osteoarthritis, and neuropathy [17,21,46,47]. Topical capsaicin may reduce pain and inhibit cutaneous 
hypersensitivity through nociceptor fiber defunctionalization based on an in vitro study [48]. Defunctionalization occurs through continued capsaicin applications, which may result in decreased responsiveness to noxious stimuli [49]. Additionally, capsaicin is believed to have anti-inflammatory analgesic effects $[47,48]$.

In one meta-analysis involving two studies $[17,21]$ in this systematic review, capsaicin cream versus placebo showed no main treatment effect in terms of TMJ-associated pain. In one study, joint sensitivity to palpation on the affected side disappeared in 4 of 15 patients in the capsaicin group and in 3 of 13 patients in the placebo group by the end of the experiment [21]. In patients with chronic soft tissue pain, patients receiving $0.05 \%$ topical capsaicin had diminished pain scores than those receiving placebo [47]. The efficacy of capsaicin in treating TMD-associated pain remains equivocal $[17,21]$. Topical application of capsaicin often results in burning or itching sensation [46,48]. Defunctionalization occurs through continued applications, which may result in decreased responsiveness to noxious stimuli $[47,48]$. Further studies are needed to confirm this.

\section{5) Ping On cream}

A topical medication, Ping On cream, consisting of Chinese herbs (natural camphor, menthol, peppermint oil, birch oil, eucalyptus oil, sandalwood oil, bee wax, and aromatic oil) along with petroleum jelly, was reported to reduce both TMJ- and muscle-related pain in one RCT [2]. Birch oil, which also consists of methyl salicylate, has anti-inflammatory properties. The ethanolic extract of propolis (bee wax) has also been proposed to have anti-inflammatory effects in rats [50]. Menthol may produce analgesic effects by activating TRPM8, heat-activated TRPV3, and inhibiting TRPA1 as well as desensitizing TRPM8 [51].

$\mathrm{Li}$ et al. [2] showed statistically significant improvements in VAS pain score with Ping On ointment of 20 units compared to placebo at day 30 ( $\mathrm{P}<0.001)$. However, the improvement of 6 units at day 7 was not statistically significant $(P>0.05)$. Further studies are needed to confirm these findings. No previous studies have been conducted specifically with Ping On ointment in the treatment of TMD.

\section{6) Bee venom}

Bee venom, also known as apipuncture, has been utilized for arthritic pain [52]. Bee venom, or apitoxin, consists of a mixture of proteins, including adolapin, which is a COX inhibitor and thus has anti-inflammatory and analgesic properties [53]. According to the authors, the use of bee venom [2] versus placebo resulted in changes in VAS score after 2 weeks of treatment, which were statistically significant and favorable to bee venom ( $\mathrm{P}<0.001)$; however, we could not conduct a meta-analysis as the standard deviations of the VAS pain score were not reported. Prior reviews did not discuss the results of bee venom therapy in the context of TMD-associated pain relief. Lee et al. [54] reported that bee venom acupuncture helped in the treatment of arthritis; however, the evidence is limited. According to a systematic review by Lee at al. [55], one study reported a significant reduction in morning stiffness and joint swelling and tenderness, and an improvement in the quality of life of patients with RA. However, the evidence is of low quality, as it is based on the results of only one RCT. Although the bee venom is used to treat RA and other neurological conditions, bee venom therapy may have some adverse effects, including local swelling or itching [56]. More studies are needed to investigate the effect of topical bee venom for TMD treatment in humans.

\section{7) $\mathrm{CBD}$}

CBD and tetrahydrocannabinol (THC) are active components of Cannabis sativa L. Wong et al. found that the trigeminal ganglion had both $\mathrm{CB} 1$ and $\mathrm{CB} 2$ receptors that innervate the rat masseter muscles and proposed that these receptors are involved in analgesia [57]. In this review, the improvement in VAS pain score of 34 units from baseline (0-100) at day 14 was significantly more favorable to topical CBD compared to placebo (P < 
0.001). Transdermal cannabinoids have shown efficacy in reducing pain and inflammation in mice [58]. Animal models have shown that topical CBD application has a potential for pain relief as well as pain-related behaviors and inflammation, with no evident side effects [59]. A recent study that reviewed the efficacy of $\mathrm{CBD}$ in the treatment of joint disease and orthopedic joint replacement found no approved pharmaceutical products that contain only CBD for pain [60]. Further prospective placebo-controlled studies to investigate the effect of topical CBD for treating TMD in humans are needed.

\section{Overall completeness and applicability of evidence}

The electronic databases searched were Cochrane Library, Medline via PubMed, EMBASE, and Web of Science, up to February 7, 2020; the search was limited to English published articles. We manually searched the reference sections and included studies to identify additional studies. The results of this systematic review are applicable to TMD patients aged 18-61 years. Since the reported treatment duration ranged from $2 \mathrm{~d}$ to 90 $\mathrm{d}$, this review does not address the long-term efficacy of topical interventions. These results are applicable to both genders and Europeans [18,20,24], North Americans $[17,19,23]$, and Asians [2,21,22].

\section{Heterogeneity of the review and analysis of biases}

In this review, it was evident that there was clinical heterogeneity in multiple areas. Owing to the different interventions applied in the study group, subgroup analyses comparing topical NSAIDs versus placebo, systemic versus topical NSAIDs, and plant- or animalbased nutraceuticals versus placebo were performed separately. The population selected was heterogeneous, except for two studies that included only female patients $[17,23]$, which could lead to a bias in the results if the response to the intervention differed between female and male patients. There is also a heterogeneity in the treatment and follow-up durations; one trial had a follow-up duration of $2 \mathrm{~d}$ [22] and another had the longest follow-up after treatment of $90 \mathrm{~d}$ [23]. Some of the studies allowed the patients to use medications, such as extra strength acetaminophen, [23] or continued analgesic treatment [2], during the trial that could bias the results. Additionally, heterogeneity was observed in the topical intervention's application methods; the treatment method in two studies included application of ultrasound massage [22] or a simple massage to the masseter muscles [20], which could bias the results, if the massage affected the patients' response to the topical intervention.

\section{Implications for research}

This systematic review and meta-analyses demonstrated that topical medications had low evidence in reducing pain associated with TMD due to its high or unclear risk of bias. The quality of the evidence was low due to the unclear blinding and small sample size of the subjects in each meta-analysis. The studies did not mention any funding sources. The meta-analyses highlight the need for future well-designed, double-blinded randomized, placebo-controlled studies with larger sample sizes of patients receiving NSAIDs, Ping On, CBD, and bee venom. Future studies should also explore other topical agents that may be useful in reducing pain, such as menthol and other compound medications. In addition, the efficacy of higher doses of NSAIDs and other topical interventions as well as their duration of use, compliance rate, and possible side effects need further investigation.

\section{Implications for clinical practice}

Utilizing topical interventions for patients who are pharmacophobic or who may develop side effects from systemic interventions may be considered as an adjunctive treatment, as this treatment method results in minimal resorption of the drug into the blood stream and mild side effects. Nevertheless, caution should be considered in patients who might develop severe side effects from topical agents, which could be avoided by always testing a small skin area before starting treatment.

\section{Conclusions}

There is not enough evidence to support the use of 
topical NSAID and capsaicin cream to palliate pain in patients with TMD, and limited evidence was found for bee venom, Ping On, CBD, and Theraflex-TMJ topical cream. The low quality of the evidence was due to the unclear or high risk of bias and the limited number of studies for each intervention. Additional studies with minimal risk of bias and larger numbers of participants are needed to confirm these results.

\section{AUTHOR ORCOIS}

Mariam Mena: https://orcid.org/0000-0002-8225-9539

Lana Dalbah: https://orcid.org/0000-0002-1184-9590

Lauren Levi: https://orcid.org/0000-0002-3797-2255

Mariela Padilla: https://orcid.org/0000-0003-3078-2544

Reyes Enciso: https://orcid.org/0000-0003-1751-3286

\section{AUHOR GONLRUIIONS}

Mariam Mena: Conceptualization, Investigation, Methodology, Writing - original draft, Writing - review \& editing

Lana Dalbah: Conceptualization, Investigation, Methodology, Writing - original draft, Writing - review \& editing

Lauren Levi: Conceptualization, Investigation, Methodology, Writing - original draft, Writing - review \& editing

Mariela Padilla: Conceptualization, Methodology, Writing - original draft, Writing - review \& editing

Reyes Enciso: Conceptualization, Formal analysis, Investigation, Methodology, Validation, Writing - original draft, Writing - review \& editing

ACKNOWLEDGMENTS: The protocol was sent to PROSPERO on December 1, 2019 and published on July 21, 2020 with registration \# CRD42020160723.

DECLARATION OF INTERESTS: The authors have no conflicts of interest to declare.

FUNDING: The authors have not received funding for this work.

\section{REFERENCES}

1. Okeson JP. Current terminology and diagnostic classification schemes. Oral Surg Oral Med Oral Pathol Oral Radiol Endod 1997; 83: 61-4.
2. Li LCF, Wong RWK, Rabie ABM. Clinical effect of a topical herbal ointment on pain in temporomandibular disorders: a randomized placebo-controlled trial. J Altern Complement Med 2009; 15: 1311-7.

3. Magalhães BG, Freitas JLDM, Barbosa ACDS, Gueiros, MCSN, Gomes SGF, Rosenblatt A, et al. Temporomandibular disorder: otologic implications and its relationship to sleep bruxism. Braz J Otorhinolaryngol 2018; 84: 614-9.

4. Litt MD, Porto FB. Determinants of pain treatment response and nonresponse: identification of TMD patient subgroups. J Pain 2013; 14: 1502-13.

5. Clark GT. Classification, causation and treatment of masticatory myogenous pain and dysfunction. Oral Maxillofac Surg Clin North Am 2008; 20: 145-57.

6. Goulet JP. Contemporary approach to the nonsurgical management of temporomandibular disorders. Alpha Omegan 2003; 96: 47-56.

7. Butts R, Dunning J, Pavkovich R, Mettille J, Mourad F. Conservative management of temporomandibular dysfunction: a literature review with implications for clinical practice guidelines (Narrative review part 2). J Bodyw Mov Ther 2017; 21: 541-8.

8. Schiffman E, Ohrbach R, Truelove E, Look J, Anderson G, Goulet JP, et al. Diagnostic Criteria for temporomandibular disorders (DC/TMD) for clinical and research applications: recommendations of the international RDC/TMD consortium network* and orofacial pain special interest group. J Oral Facial Pain Headache 2014; 28: 6-27.

9. International Classification of Orofacial Pain, 1st edition (ICOP). Cephalalgia 2020; 40: 129-221.

10. Dionne RA. Pharmacologic treatments for temporomandibular disorders. Oral Surg Oral Med Oral Pathol Oral Radiol Endod 1997; 83: 134-42.

11. Funk CD, FitzGerald GA. COX-2 inhibitors and cardiovascular risk. J Cardiovasc Pharmacol 2007; 50: 4709.

12. Maniar KH, Jones IA, Gopalakrishna R, Vangsness CT. Lowering side effects of NSAID usage in osteoarthritis: recent attempts at minimizing dosage. Expert Opin 
Pharmacother 2018; 19: 93-102.

13. Sheer R, Schwab P, Essex MN, Cappelleri JC, Reiners A, Bobula J, et al. Factors related to the use of topical vs. oral NSAIDs for sprains, strains, and contusions in a senior population: a retrospective analysis of administrative claims data. Drugs Aging 2018; 35: 937-50.

14. Altman R, Barkin RL. Topical therapy for osteoarthritis: clinical and pharmacologic perspectives. Postgrad Med 2009; 121: 139-47.

15. Senye M, Mir CF, Morton S, Thie NM. Topical nonsteroidal anti-inflammatory medications for treatment of temporomandibular joint degenerative pain: a systematic review. J Orofac Pain 2012; 26: 26-32.

16. Vaile JH, Davis P. Topical NSAIDs for musculoskeletal conditions: a review of the literature. Drugs 1998; 56: 783-99.

17. Campbell BK, Fillingim RB, Lee S, Brao R, Price DD, Neubert JK. Effects of high-dose capsaicin on TMD subjects: a randomized clinical study. JDR Clin Transl Res 2017; 2: 58-65.

18. Di Rienzo Businco L, Di Rienzo Businco A, D’Emilia M, Lauriello M, Coen Tirelli G. Topical versus systemic diclofenac in the treatment of temporo-mandibular joint dysfunction symptoms. Acta Otorhinolaryngol Ital 2004; 24: $279-83$.

19. Lobo SL, Mehta N, Forgione AG, Melis M, Al-Badawi E, Ceneviz C, et al. Use of Theraflex-TMJ topical cream for the treatment of temporomandibular joint and muscle pain. Cranio 2004; 22: 137-44.

20. Nitecka-buchta A, Buchta P, Tabeńska-Bosakowska E, Walczyńska-Dragoń K, Baron S. Myorelaxant effect of bee venom topical skin application in patients with RDC/TMD Ia and RDC/TMD Ib: a randomized, double blinded study. Biomed Res Int 2014; 2014: 1-9.

21. Winocur E, Gavish A, Halachmi M, Eli I, Gazit E. Topical application of capsaicin for the treatment of localized pain in the temporomandibular joint area. J Orofac Pain 2000; 14: $31-6$.

22. Shin SM, Choi JK. Effect of indomethacin phonophoresis on the relief of temporomandibular joint pain. Cranio 1997; 15: $345-8$.
23. Wilson FM. Thesis, master of science in medical sciences-dentistry. Edmonton, Canada, University of Alberta. 2008.

24. Nitecka-buchta A, Nowak-Wachol A, Wachol K, Walczyńska-Dragon $\mathrm{K}$, Olczyk $\mathrm{P}$, Batoryna $\mathrm{O}$, et al. Myorelaxant effect of transdermal cannabidiol application in patients with TMD: a randomized, double-blind trial. J Clin Med 2019; 8: 1-17, 21.

25. Moher D, Liberati A, Tetzlaff J, Altman DG. Preferred reporting items for systematic reviews and meta-analyses: the PRISMA statement. PLoS Med 2009; 6: e1000097.

26. Higgins J, Green S. Cochrane handbook for systematic reviews of interventions version 5.1.0 [Updated March 2011].; 2011.

Available from http://www.cochrane-handbook.org.

27. Cochran WG. The combination of estimates from different experiments. Biometrics 1954; 10: 101-29.

28. Higgins JPT, Thompson SG. Quantifying heterogeneity in a meta-analysis. Stat Med 2002; 21: 1539-58.

29. Cashman JN. The mechanisms of action of NSAIDs in analgesia. Drugs 1996; 52: 13-23.

30. Lanas A. Nonsteroidal antiinflammatory drugs and cyclooxygenase inhibition in the gastrointestinal tract: a trip from peptic ulcer to colon cancer. Am J Med Sci 2009; 338: 96-106.

31. Kelly AM. The minimum clinically significant difference in visual analogue scale pain score does not differ with severity of pain. Emerg Med J 2001; 18: 205-7.

32. Kassan DG, Lynch AM, Stiller MJ. Physical enhancement of dermatologic drug delivery: iontophoresis and phonophoresis. J Am Acad Dermatol 1996; 34: 657-66.

33. Newman JT, Nellermoe MD, Carnett JL. Hydrocortisone phonophoresis a literature review. J Am Podiatr Med Assoc 1969; 10: 57-9.

34. Ramakrishnan SN, Aswath N. Comparative efficacy of analgesic gel phonophoresis and ultrasound in the treatment of temporomandibular joint disorders. Indian J Dent Res 2019; 30: 512-5.

35. Calesnick B. Current status of dimethyl sulfoxide (DMSO). Am Fam Physician 1981; 23: 167-8.

36. Conforti A, Franco L, Menegale GP, Milanino R, Piemonte 
G, Velo G. Serum copper and ceruloplasmin levels in rheumatoid arthritis and degenerative joint disease and their pharmacological implications. Pharmacol Res Commun 1983; 15: 859-67.

37. Sorenson JR. Copper complexes - a unique class of anti-arthritic drugs Prog Med Chem 1978; 15: 211-60.

38. Milanino R, Conforti A, Franco L, Marrella M, Velo G. Copper and inflammation - a possible rationale for the pharmalogical manipulation of inflammatory discorders. Agents Actions 1985; 16: 504-13.

39. Salgueiro MJ, Zubillaga M, Lysionek A, Cremaschi G, Goldman CG, Caro R, et al. Zinc status and immune system relationship: a review. Biol Trace Elem Res 2000; 76: 193-205.

40. Fraker PJ, Gershwin ME, Good RA, Prasad A. Interrelationships between zinc and immune function. Fed Proc 1986; 45: 1474-9.

41. Gupta M, Mahajan VK, Mehta KS, Chauhan PS. Zinc therapy in dermatology: a review. Dermatol Res Pract 2014; 2014: 709152.

42. Sorenson JR, Hangarter W. Treatment of rheumatoid and degenerative diseases with copper complexes - a review with emphasis on copper-salicylate. Inflammation 1977; 2: $217-38$.

43. Seth BL. Comparative pharmacokinetics and bioavailability study of percutaneous absorption of diclofenac from two topical formulations containing drug as a solution gel or as an emulsion gel. Arzneimittelforschung 1992; 42: 120-2.

44. Heyneman CA. Topical nonsteroidal antiinflammatory drugs for acute soft tissue injuries. Ann Pharmacother 1995; 29: $780-2$.

45. Hsieh LF, Hong CZ, Chern SH, Chen CC. Efficacy and side effects of diclofenac patch in treatment of patients with myofascial pain syndrome of the upper trapezius. J Pain Symptom Manage 2010; 39: 116-25.

46. Lotz M. Experimental models of arthritis: Identification of substance $\mathrm{P}$ as a therapeutic target and use of capsaicin to manage joint pain and inflammation. Semin Arthritis Rheum 1994; 23: 10-7.

47. Chrubasik S, Weiser T, Beime B. Effectiveness and safety of topical capsaicin cream in the treatment of chronic soft tissue pain. Phytother Res 2010; 24: 1877-85.

48. Wang XR, Gao SQ, Niu XQ, Li LJ, Ying XY, Hu ZJ, et al. Capsaicin-loaded nanolipoidal carriers for topical application: design, characterization, and in vitro/in vivo evaluation. Int J Nanomedicine 2017; 12: 3881-98.

49. Malmberg AB, Mizisin AP, Calcutt NA, Von Stein T, Robbins WR, Bley KR. Reduced heat sensitivity and epidermal nerve fiber immunostaining following single applications of a high-concentration capsaicin patch. Pain 2004; 111: 360-7.

50. Park EH, Kahng JH. Suppressive effects of propolis in rat adjuvant arthritis. Arch Pharm Res 1999; 22: 554-8.

51. Patel T, Ishiuji Y, Yosipovitch G. Menthol: A refreshing look at this ancient compound. J Am Acad Dermatol 2007; 57: $873-8$.

52. Son DJ, Lee JW, Lee YH, Song HS, Lee CK, Hong JT. Therapeutic application of anti-arthritis, pain-releasing, and anti-cancer effects of bee venom and its constituent compounds. Pharmacol Ther 2007; 115: 246-70.

53. Shkenderov S, Koburova K. Adolapin - a newly isolated analgetic and anti-inflammatory polypeptide from bee venom. Toxicon 1982; 20: 317-21.

54. Lee JD, Park HJ, Chae Y, Lim S. An overview of bee venom acupuncture in the treatment of arthritis. Evid Based Complement Alternat Med 2005; 2: 79-84.

55. Lee JA, Son MJ, Choi J, Jun JH, Kim JI, Lee MS. Bee venom acupuncture for rheumatoid arthritis: a systematic review of randomised clinical trials. BMJ Open 2014; 4: e006140.

56. Zhang S, Liu Y, Ye Y, Wang XR, Lin LT, Xiao LY, et al. Bee venom therapy: potential mechanisms and therapeutic applications. Toxicon 2018; 148: 64-73.

57. Wong H, Cairns BE. Cannabidiol, cannabinol and their combinations act as peripheral analgesics in a rat model of myofascial pain. Arch Oral Biol 2019; 104: 33-9.

58. Lodzki M, Godin B, Rakou L, Mechoulam R, Gallily R, Touitou E. Cannabidiol - transdermal delivery and antiinflammatory effect in a murine model. J Control Release 2003; 93: 377-87.

59. Hammell DC, Zhang LP, Ma F, Abshire SM, McIlwrath SL, Stinchcomb AL, et al. Transdermal cannabidiol reduces 
inflammation and pain-related behaviours in a rat model of arthritis. Eur J Pain 2016; 20: 936-48.

60. Gusho CA, Court T. Cannabidiol: a brief review of Its therapeutic and pharmacologic efficacy in the management of joint disease. Cureus 2020; 12: e7375.

61. Dworkin SF, LeResche L. Research diagnostic criteria for temporomandibular disorders: review, criteria, examinations and specifications, critique. J Craniomandib Disord 1992; 6: 301-55.
62. Osiewicz MA, Lobbezoo F, Loster BW, Wilkosz M, Naeije M, Ohrbach R. Research diagnostic criteria for temporomandibular disorders (RDC/TMD) - the polish version of a dual-axis system for the diagnosis of TMD. J Stomatol 2013; 66: 576-649.

63. Collins SL, Moore RA, McQuay HJ. The visual analogue pain intensity scale: what is moderate pain in millimetres? Pain 1997; 72: 95-7. 